\title{
Human milk extracellular vesicles target nodes in interconnected signalling pathways that enhance oral epithelial barrier function and dampen immune responses
}

\author{
Marijke I. Zonneveld ${ }^{1,2,{ }^{*}} \quad$ Martijn J.C. van Herwijnen ${ }^{1, *} \quad \mid \quad$ Marcela M. Fernandez-Gutierrez ${ }^{3, \#}$ \\ Alberta Giovanazzi $^{1, \# ~ \mid ~ A n n e ~ M a r i t ~ d e ~ G r o o t ~}{ }^{4, \# ~ \mid ~ M a r i j e ~ K l e i n j a n ~}{ }^{1} \quad$ Toni M.M. van Capel ${ }^{5}$ \\ Alice J.A.M. Sijts ${ }^{4} \quad$ Leonie S. Taams ${ }^{6} \quad$ Johan Garssen ${ }^{2,7} \quad$ Esther C. de Jong \\ Michiel Kleerebezem $^{3} \quad$ | Esther N.M. Nolte-'t Hoen ${ }^{1,+} \quad$ Frank A. Redegeld ${ }^{2,+}$ | \\ Marca H.M. Wauben ${ }^{1}$ \\ ${ }^{1}$ Department of Biomolecular Health Sciences, Faculty of Veterinary Medicine, Utrecht University, Utrecht, The Netherlands \\ ${ }^{2}$ Division of Pharmacology, Department of Pharmaceutical Sciences, Faculty of Science, Utrecht University, Utrecht, The Netherlands \\ ${ }^{3}$ Host-Microbe Interactomics Group, Department of Animal Sciences, Wageningen University, Wageningen, The Netherlands \\ ${ }^{4}$ Division of Infectious Diseases \& Immunology, Department of Biomolecular Health Sciences, Faculty of Veterinary Medicine, Utrecht University, Utrecht, The Netherlands \\ ${ }^{5}$ Department of Experimental Immunology, Academic Medical Center, Amsterdam, The Netherlands Centre for inflammation, University of AmsterdamAmsterdam Infection \& \\ Immunity Institute (AI\&II), Amsterdam, The Netherlands \\ ${ }^{6}$ Centre for Inflammation Biology and Cancer Immunology, Department of Inflammation Biology, School of Immunology \& Microbial Sciences, King's College London, \\ London, UK \\ ${ }^{7}$ Global Centre of Excellence Immunology, Danone Nutricia Research, Utrecht, The Netherlands
}

\section{Correspondence}

Marca H.M. Wauben, Department of Biomolecular Health Sciences, Faculty of Veterinary Medicine, Utrecht University, Utrecht, The Netherlands. Email:M.H.M.Wauben@uu.nl

\section{${ }^{*}$ Marijke I. Zonneveld and Martijn J.C. van} Herwijnen are shared 1st authors.

\# Marcela M. Fernandez-Gutierrez, Alberta Giovanazzi, and Anne Marit de Groot are shared 2nd authors.

+Esther N.M. Nolte-'t Hoen and Frank A. Redegeld are shared 2 nd senior authors.

\section{Funding information}

TIFN Food and nutrition; Stichting voor de Technische Wetenschappen, Grant/Award Number: 11676; H2020 European Research Council, Grant/Award Number: 442337581; H2020 Marie Skłodowska-Curie Actions, Grant/Award Number: 722148; H2020 Future and Emerging Technologies, Grant/Award Number: 801367

\begin{abstract}
Maternal milk is nature's first functional food. It plays a crucial role in the development of the infant's gastrointestinal (GI) tract and the immune system. Extracellular vesicles (EVs) are a heterogeneous population of lipid bilayer enclosed vesicles released by cells for intercellular communication and are a component of milk. Recently, we discovered that human milk EVs contain a unique proteome compared to other milk components. Here, we show that physiological concentrations of milk EVs support epithelial barrier function by increasing cell migration via the p38 MAPK pathway. Additionally, milk EVs inhibit agonist-induced activation of endosomal Toll like receptors TLR3 and TLR9. Furthermore, milk EVs directly inhibit activation of CD4+ T cells by temporarily suppressing $\mathrm{T}$ cell activation without inducing tolerance. We show that milk EV proteins target key hotspots of signalling networks that can modulate cellular processes in various cell types of the GI tract.
\end{abstract}

\section{KE YWORDS}

breast milk, exosomes, extracellular vesicles, gastrointestinal tract, human milk, immune modulation, immune system development, oral cavity, T cell modulation, TLR modulation

This is an open access article under the terms of the Creative Commons Attribution License, which permits use, distribution and reproduction in any medium, provided the original work is properly cited.

(C) 2021 The Authors. Journal of Extracellular Vesicles published by Wiley Periodicals, LLC on behalf of the International Society for Extracellular Vesicles 
In the neonate, the epithelial barrier of the GI tract needs to grow and mature, while the adaptive immune system is still developing (Renz et al., 2011). This requires cellular regulation and education which, in part, is induced by components in mother's milk. Although the importance of breastfeeding is widely recognized, the milk components and molecular processes involved in these developmental processes remain largely obscure (Victora et al., 2016). This limited understanding is mainly due to the complexity of milk as it is composed of a wide range of bioactive macromolecular structures with partially overlapping physical properties (Ballard \& Morrow, 2013; Chatterton et al., 2013; van Herwijnen et al., 2016). Among these components are extracellular vesicles (EVs) (Admyre et al., 2007; van Herwijnen et al., 2016; Zonneveld et al., 2014), which are nanosized particles released by cells. The release of EVs and the incorporation of molecular cargo into EVs is tightly regulated by the producing cell, resulting in a great heterogeneity of EVs specifically tailored for targeted intercellular communication (Mathieu et al., 2019; Robbins \& Morelli, 2014). Altogether this contributes to the versatile capacity of EVs to modulate cellular responses at multiple levels. The multifaceted nature of EVs allows for the fine-tuning of complex signalling pathways in order to control the magnitude, kinetics and duration of cellular responses (Raposo \& Stahl, 2019; Steenbeek et al., 2018). Previously, we have established an isolation procedure specifically designed for the reliable isolation of EVs from human milk (Zonneveld et al., 2014) and performed indepth proteomics analysis of milk EVs (van Herwijnen et al., 2016). We discovered several milk EV-associated proteins not identified before in human milk, that potentially contribute to both the development of the epithelial barrier and maintenance of immune homeostasis (van Herwijnen et al., 2016). In this study, we explored the physiological role of milk EVs in these processes and linked their protein cargo to underlying signalling pathways.

In order to isolate pure milk EVs we removed cells and cream from fresh (non-frozen) breast milk before biobanking. We then used differential centrifugation followed by density gradient separation and Size Exclusion Chromatography (SEC) to obtain EVs in the desired culture medium for downstream analysis (Figure 1a). Importantly, the concentration of EVs in the final sample was comparable to that of EVs in milk, allowing us to study EVs in their physiological concentration. The milk matrix also contains several non-EV components that can influence cells of the GI tract and might be co-isolated.

Additionally, our isolation procedure might introduce artefacts affecting cellular read-out systems. Therefore, we also prepared a donor-matched procedural control, called EV-depleted, to validate any EV-mediated effects (Figure 1a). To quantify the amount of EVs, we performed nanoparticle tracking analysis (NTA) and observed a substantial number of particles in the EV samples (average $9.3 \times 10^{10} \pm 1.2 \times 10^{10}$ particles/ml; Figure $1 b$ ). In contrast, the EV-depleted controls contained much less particles with fewer valid tracks and altered light scattering properties (Supplementary File 1 and Supplementary Figure 1). The size distribution of the isolated milk EVs ranged between 199-220 nm, albeit differences between the individual samples were observed, reflecting the heterogeneity of the milk EV population (Figure 1b). Next, we confirmed the presence of EV-associated markers CD9, CD63, Flotillin-1 and HSP70 in the milk EVs by Western blot analysis, while these markers were undetectable in the EV-depleted controls (Figure 1c). In contrast, lactoferrin, an abundant soluble non-EV milk protein was detected in both EV samples and their matched EV-depleted controls, indicating the presence of milk matrix proteins in the procedural controls. Interestingly, although milk composition changes during lactation and can differ between mothers, we demonstrate rather limited inter-donor variation in the EV characteristics. The might be due to the use of mature milk samples (average of $7.2 \pm 3$ months post-partum) in which the variation in overall milk composition between mothers has becomes less (Ballard \& Morrow, 2013).

Although often overlooked, the first interaction of milk components with the infant's mucosa occurs in the oral cavity, a site that needs to maintain an intact epithelial barrier and contains mucosa-associated lymphoid tissue (MALT) (Groeger \& Meyle, 2019; Moutsopoulos \& Konkel, 2018). To determine whether milk EVs play a role in maintenance of the physical epithelial integrity, we performed a gap closure assay with gingival epithelial cells in the presence of milk EVs. Milk EVs significantly increased the re-epithelialization rate of the epithelial cells to almost the same level as the positive control TGF- $\alpha$, while EVdepleted milk supernatant did not (Figure 2a and 2b, Supplementary File 2). Re-epithelialization can occur through p38 MAPKdependent migration (Huang, 2004; Sharma et al., 2003), either or not combined with cell proliferation through MEK-ERK signalling (Mebratu \& Tesfaigzi, 2009). To assess which milk EV proteins could be involved in the enhanced re-epithelialization and which pathways were likely targeted, we performed enrichment, network- and functional annotation analysis on the milk EV proteome (van Herwijnen et al., 2016). Using enrichment analysis, we first identified 33 significantly enriched GO-terms involved in cell cycle and migration to which collectively 159 proteins were associated (Supplementary File 3). Interestingly, network analysis revealed that 134 of these 159 proteins can form protein-protein interactions (Supplementary Figure 2A), indicating that the combined EV cargo can be delivered as protein networks. Finally, functional annotation analysis was performed to link each protein from the identified protein networks to relevant signalling cascades involved in re-epithelization, while also visualizing the expected mode of action of the milk EV protein (Supplementary Figure 2B). These analyses resulted in a model that shows that milk EV proteins can interact at multiple levels in signalling cascades and unveils several 'hot spots' where milk EV proteins formed nodes with a high number of potential interactions. Several of these nodes are formed around key cellular proteins that are involved in either inhibition of cell cycle (and proliferation), or stimulation of migration via p38 MAPK downstream of RAC1 and CDC42 (Supplementary Figure 2B and summarized in Figure 2c). To investigate whether milk EVs primarily affected re-epithelialization by stimulating migration, cells were cultured in the presence of pharmacological inhibitors of p38 MAPK or MEK1/2 as a control for proliferation. Inhibition of p38 MAPK abolished the increased re-epithelialization rate induced by EVs (Figure 2d), while MEK1/2 inhibition showed only a minor reduction of EV-mediated re-epithelialization (Figure 2e). To 
a

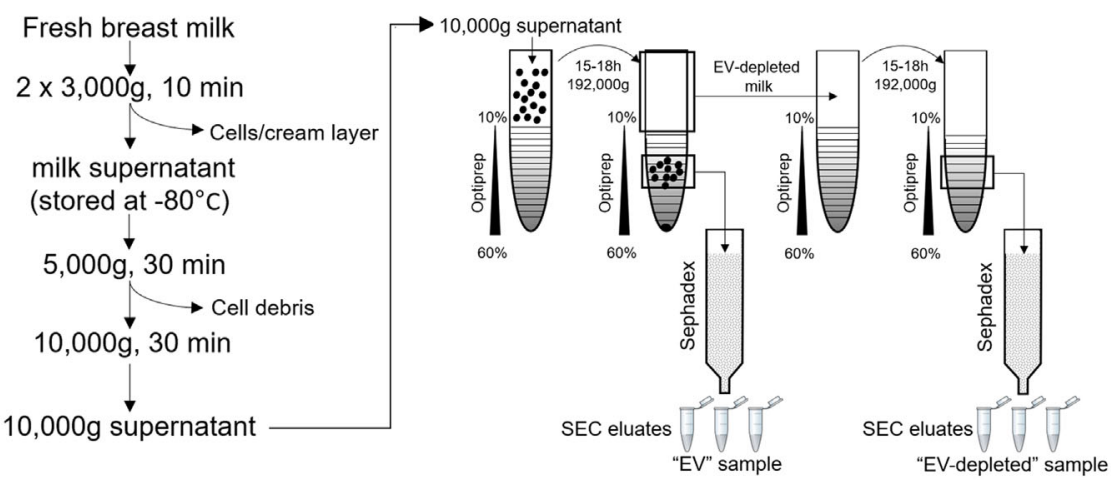

b
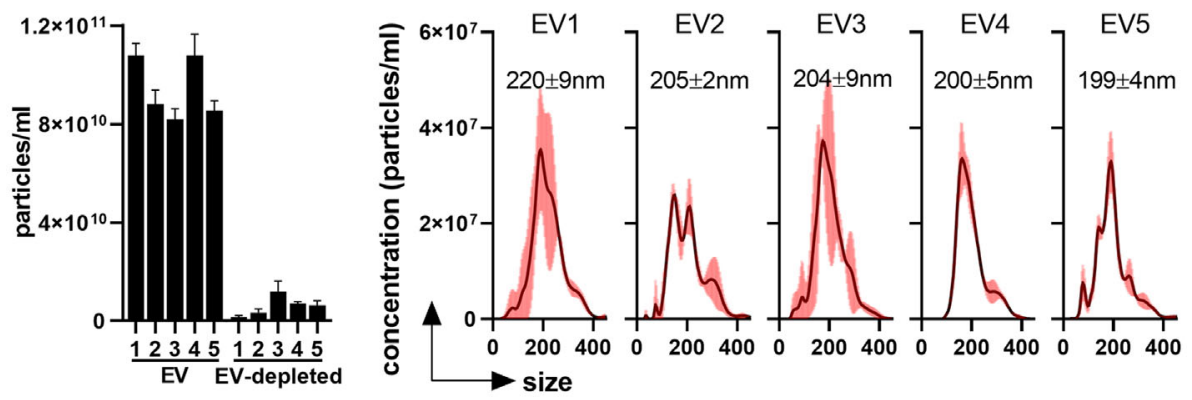

C

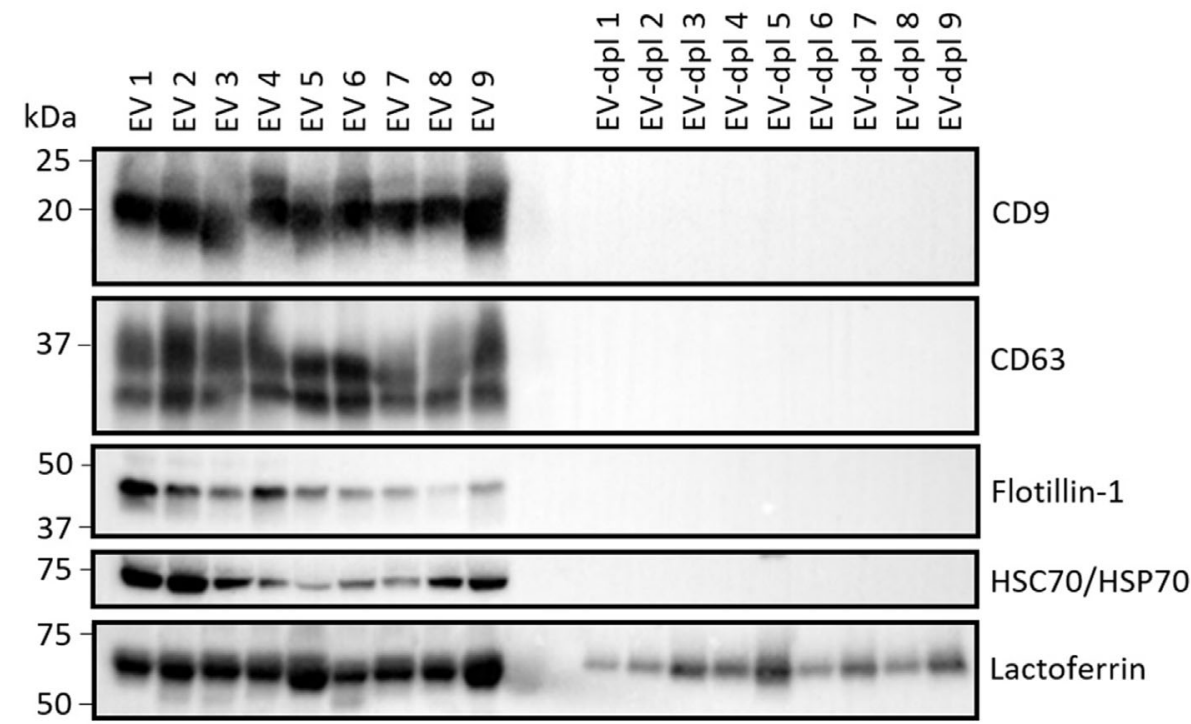

FI G U RE 1 Isolation and characterization of human milk EVs and EV-depleted milk control. [a) Schematic overview of the isolation of milk EVs and the EV-depleted milk control using differential centrifugation followed by density gradient ultracentrifugation. The original isolation procedure (Zonneveld et al., 2014) applied sucrose as a density medium. However, to maintain functionality of isolated EVs, Optiprep was used to build the gradient for ultracentrifugation. Size exclusion chromatography (SEC) using Sephadex was applied to separate EVs from Optiprep and to collect the 'EV fractions' in the culture medium needed for functional analysis. The concentration of EVs used in the in vitro assays were within the physiological range, since the starting volume of $10,000 \mathrm{~g}$ milk supernatant was $6.5 \mathrm{ml}$ prior to density gradient separation, while the final volume of the EV sample was $7 \mathrm{ml}$. The final volumes of EVs applied in the various assays varied between $75 \%-100 \%$ (see methods for more details). As a donor-matched procedural control, we used the EV-depleted milk supernatant from the first density gradient and applied this sample to a new density gradient after which fractions matching EV densities were collected and applied to SEC in order to obtain the final EV-depleted control. b) Concentration measurements and particle size distribution of isolated milk EVs and their respective EV-depleted controls from five donors. Flow mode measurements were performed with a NanoSight NS300 equipped with a NanoSight Syringe Pump and an optimized particles/frame rate in the range of 70-110 was used. Each measurement consisted of triplicate 60-s captures. Mean and SD of three captures are shown. Particle distribution histograms of milk EV samples show size distributions in $\mathrm{nm}$. c) The pooled SEC eluates that were used as EV or EV-depleted samples were characterized by Western blot for the presence of EV-associated markers CD9 (exposure time $1 \mathrm{~s}$ ), CD63 (exposure time $5 \mathrm{~s}$ ), Flotillin-1 (exposure time $120 \mathrm{~s}$ ), Hsp70 (exposure time $30 \mathrm{~s}$ ) and non EV-associated marker lactoferrin (exposure time $10 \mathrm{~s}$ ) (data is from 1 experiment with a single sample from nine different milk donors)] 
a

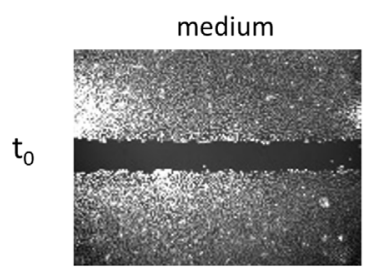

180

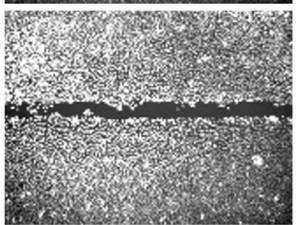

C

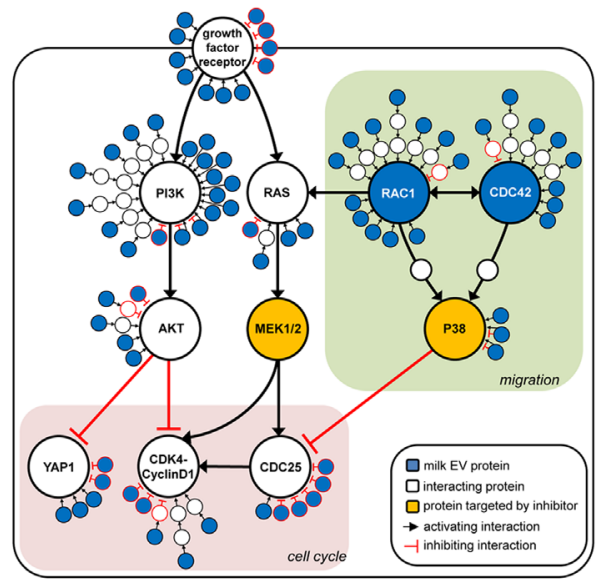

EV-depleted

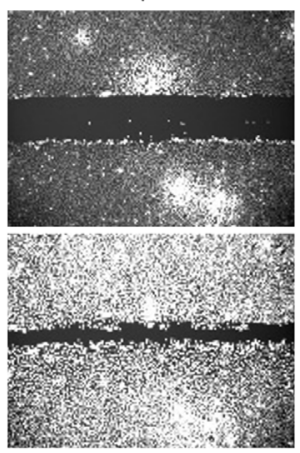

d

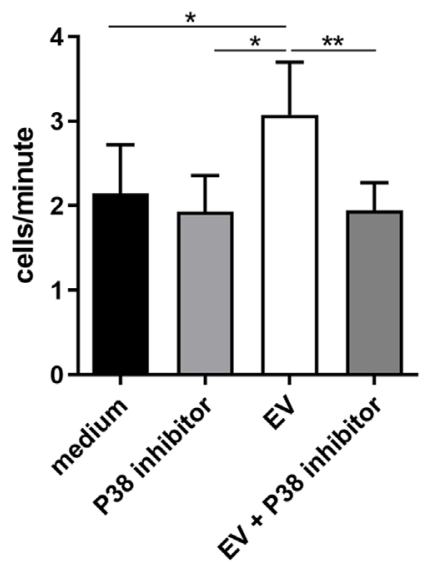

b
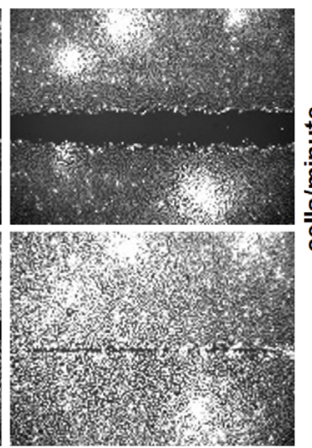

f

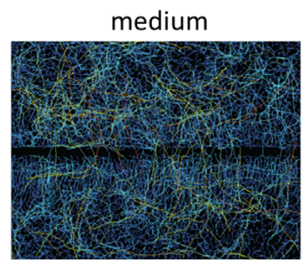

EV

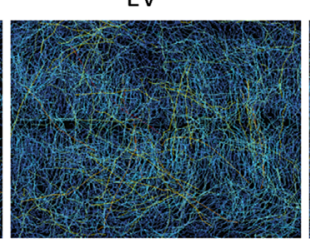

displacement (units)
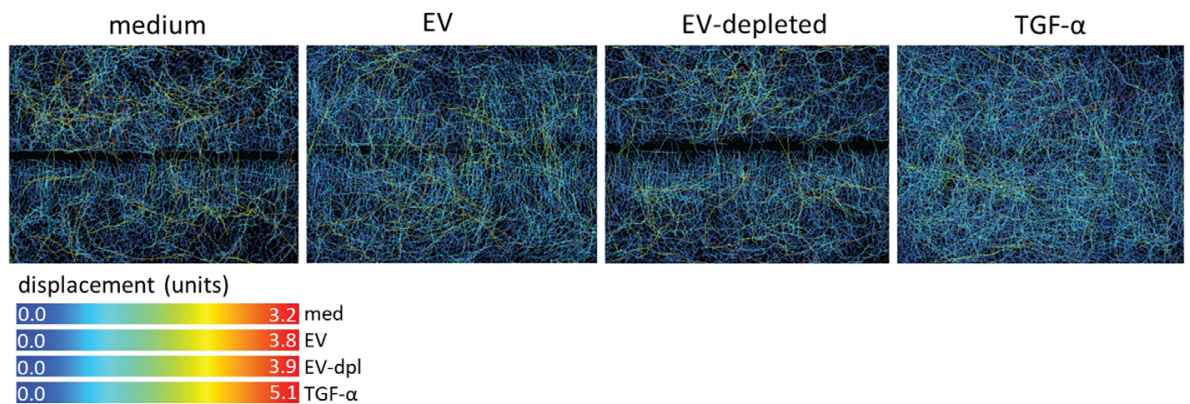

e
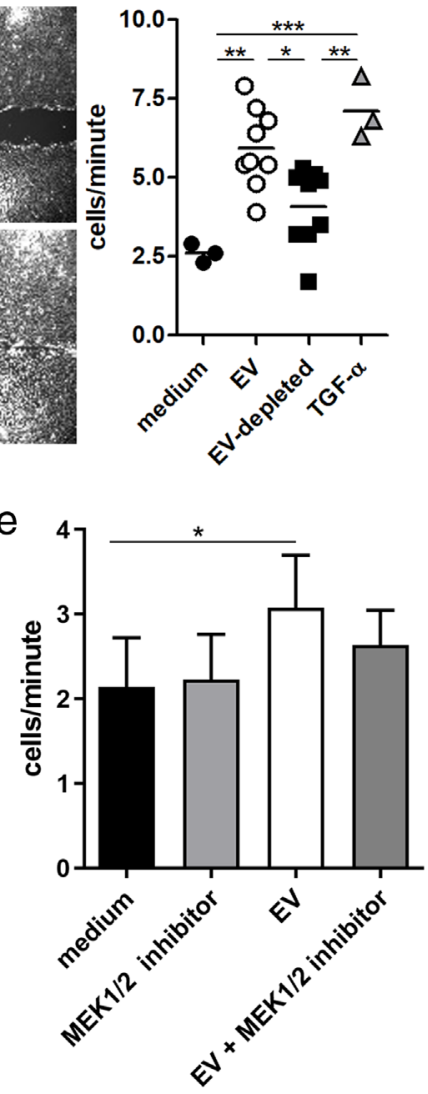

h

E-cadherin
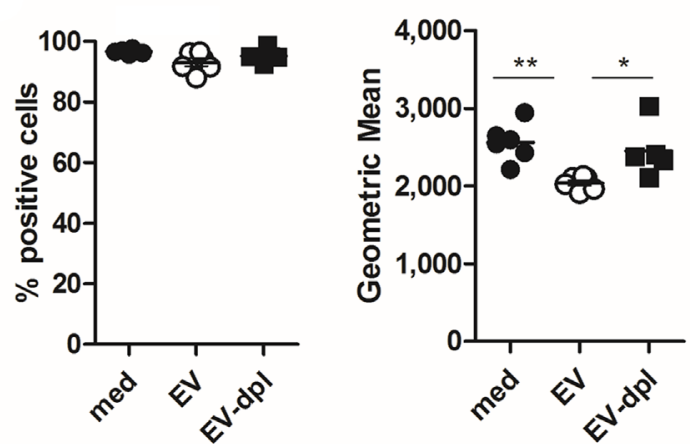

i

EPCAM
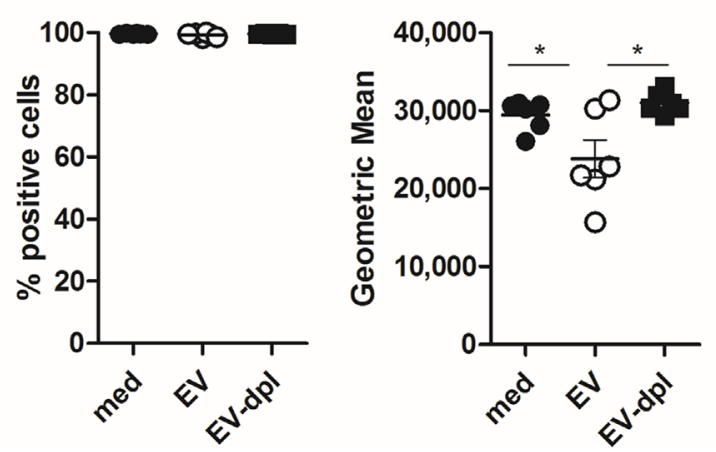

FIGURE 2 Human milk EVs enhance the formation of the epithelial barrier via p38 MAPK. [a) A gap was made in a confluent monolayer of Ca9-22 gingival epithelial cells and re-epithelialization was investigated in the presence of milk EVs, or milk donor-matched EV-depleted procedural control, or TGF$\alpha$ as a positive control. Cell migration was tracked in triplicate conditions by live cell imaging. Images were acquired every 20 min for $5 \mathrm{~h}$, or until the gap in the TGF- $\alpha$ condition was closed. Representative microscopic images of Ca9-22 cells at the start of experiment (t0) and after 180 min (t180) incubation with indicated conditions are shown. Images are representative of two experiments with three milk donors per experiment, with triplicate technical replicates. 
confirm the migratory behaviour of the epithelial cells, we performed single cell tracking to determine their displacement. In comparison to the cells cultured in medium or the EV-depleted control, exposure to milk EVs caused a significant increase in cell displacement that was characterized by cells migrating longer tracks (Figure $2 \mathrm{f}$ and $2 \mathrm{~g}$ ). As re-epithelialization is initiated instantly (Fernandez-Gutierrez et al., 2017), this process is independent of substantial de novo gene transcription or protein production and is most likely due to regulation of the existing cellular proteome. We therefore investigated rapid changes in the expression of adhesion molecules, as our model predicted the involvement of E-cadherin (hotspot in Supplementary Figure 2B) and EPCAM, which are involved in cell motility by remodelling the actin cytoskeleton (Barth et al., 2018; Shamir \& Ewald, 2015). Indeed, we show that cell surface levels of E-cadherin and EPCAM were significantly decreased in the presence of milk EVs (Figure 2h-i and Supplementary Figure 3), which would allow for faster movement of the epithelial cells.

These data also fit previous observations that p38 MAPK regulates the post-transcriptional expression of E-cadherin (Lv et al., 2011; Zohn et al., 2006). Interestingly, others have shown a role for milk EVs of rat, porcine and bovine origin in modulating intestinal epithelial cells (Chen et al., 2016; Hock et al., 2017; Yu et al., 2017), suggesting that supporting epithelial integrity could be a central and evolutionary conserved function of maternal milk EVs. This idea is further supported by the presence of conserved milk EV miRNA cargo with regulatory functions that are shared between mammalian species, which could aid in this process (Herwijnen et al., 2018). Taken together, we demonstrate that human milk EVs directly enhance gingival epithelial cell migration via p38 MAPK and cytoskeletal remodelling. Milk EV proteins likely form clusters that can target migration-related signalling cascades at multiple levels, thereby modulating the balance between key signalling nodes and tailoring cellular responses.

Epithelial cells lining the oral cavity also act as sentinels by scouting their environment via innate immune receptors. The activation of such receptors, for example, Toll-like receptors (TLRs), needs to be critically regulated in order to enable the mucosal system to appropriately defend against pathogens, but tolerate commensals (Chung \& Dale, 2008; Groeger \& Meyle, 2019; Menckeberg et al., 2015). We therefore investigated whether milk EVs could be involved in TLR regulation.

Using TLR reporter cell lines, we observed that milk EVs and the EV-depleted controls stimulated both TLR2 and TLR4 to some extent (Figure $3 \mathrm{a}$ and $3 \mathrm{~b}$ ), while TLR3 and TLR9 reporters were only triggered by their respective agonist (Figure 3c and 3d). Since milk is not sterile, and as milk collection and EV isolation might introduce microbial contaminations, we performed an endotoxin assay. We found that the milk EV samples, and to a lesser extent EV depleted controls, contained endotoxin (Supplementary Figure 4). Although it has previously been shown that EVs from other sources can stimulate TLR2 (Bretz et al., 2013), TLR3 (Seo et al., 2016), TLR4 (Bretz et al., 2013) and TLR9 (Ye et al., 2017), we show that milk EVs do not activate TLR3 and TLR9. Next, we determined whether milk EVs could regulate agonist-induced TLR activation. Both TLR2 and TLR4 reporter lines showed equal stimulation in the presence of agonist regardless of the presence of milk EVs (Figure $3 \mathrm{a}$ and $3 \mathrm{~b}$ ). In contrast, milk EVs significantly dampened the agonist-induced response of endosomal TLR3 and TLR9, while EV-depleted controls had no or reduced effects (Figure $3 \mathrm{c}$ and $3 \mathrm{~d}$ ). To assess the inhibitory effect of milk EVs on endosomal TLR activation in a more physiological setting, we evaluated their effect on gingival epithelial cells. As these epithelial cells do not express TLR9, we assessed inhibition of TLR 3 activation and found that also in these cells milk EVs reduced TLR3 activation, as measured by gene transcription of the pro-inflammatory cytokines IL6 and CXCL8 (Figure 3e). The expression profile of other TLR-related genes showed that in the presence of milk EVs, agonist-induced gene upregulation could be fully inhibited (LTA, REL, MAP2K4 and IL6), or partially inhibited (MAPK8, PELII, FOS, JUN, and CXCL8) (Figure $3 \mathrm{f}$ and Supplementary Figure 5 for complete gene array). Interestingly, the inhibition of MAP2K4 (MEK4) and of its downstream targets MAPK8 (JNK) and JUN supports the finding of epithelial cell migration via p38 MAPK (Figure 2), as MAP2K4 is predominantly involved in the activation of JNK and not p38 MAPK (Agarwal et al., 2015). In contrast to the inhibiting effects on gene expression, milk EVs enhanced the agonist-induced upregulation of EIF2AK2, as well as that of SIGIRR and SARM1, both negative regulators of TLR signalling (Kondo et al., 2012;

b) Quantification of Ca9-22 cells migrating into the gap, expressed in cells per minute during the linear growth phase. Each well was plotted as a single point and data are representative of two experiments with three milk donors in each experiment, with triplicate technical replicates. c) Schematic representation of key signalling pathways involved in gap closure. From the full annotation analysis (Supplementary file 3 and Supplementary Figure 2), key signalling pathways were selected that could be involved in gap closure. Several nodes with high interactions ( $\geq 6$ interactions) were observed. These hotspots were involved in either i) inhibition of cell cycle (e.g., YAP1, CDK4-CyclinD1 inhibition via the PI3K-AKT pathway, or CDC25 inhibition via p38 MAPK pathway), ii) activation of cell cycle (e.g., MEK1/2 via RAS), or iii) stimulation of migration (e.g., the p38 MAPK pathway via RAC1 and CDC42). Proteins present in milk EVs are depicted in blue, while interacting cellular proteins are shown in white. Key proteins involved in cell cycle or migration that were inhibited in $\mathrm{d}$ and e are highlighted in yellow. Type of interactions between proteins is shown as activating or inhibiting. $\mathrm{d}$ and e) Quantification of Ca9-22 cells migrating into the gap in the presence of p38 MAPK inhibitor (d) or MEK1/2 inhibitor (e). Bars represent mean \pm SD. Data is representative of two independent experiments with two different milk donors per experiment, with triplicate technical replicates. f) From the experiment in figure a, the image series per treatment were converted into a hyperstack and single cells were tracked using TrackMate. Total track displacement is depicted by the pseudo-coloured tracks. g) Summary of the cellular displacement per condition from figure f. h and i) Epithelial Ca9-22 cells were cultured for $5 \mathrm{~h}$ in medium or together with milk EVs or EV-depleted control after which cell surface expression of E-cadherin (h) or EPCAM was determined (i). Shown are the percentage of E-cadherin+ cells or EPCAM+ cells in the live gate and the Geometric mean of the cells positive for the respective marker. Data are representative of two experiments with three milk donors in each experiment, with a single technical replicate. Significance was calculated by Kruskal-Wallis and Dunn's multiple comparison test, one-way ANOVA and Tukey's multiple comparison test, or Brown-Forsythe and Welch ANOVA using either Games-Howell's correction for multiple comparisons with individual variances computed for each comparison or Dunnett's T3 post-test and significance was defined as ${ }^{*} P<0.05$; $^{* *} P<0.01$, ${ }^{* * *} P<0.001$, and $\left.{ }^{* * *} P<0.0001\right]$ 
a

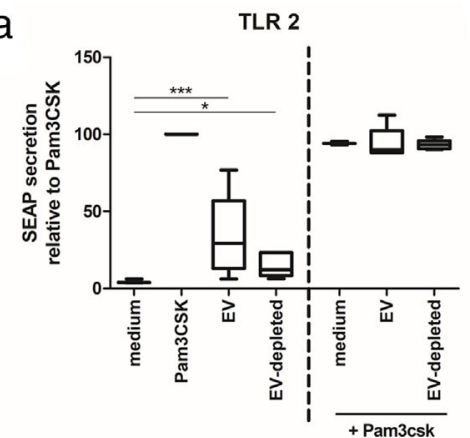

C

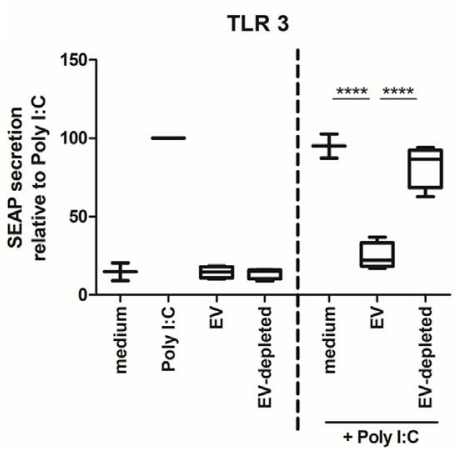

b

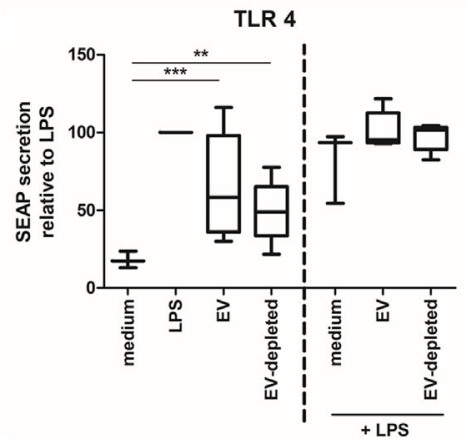

d

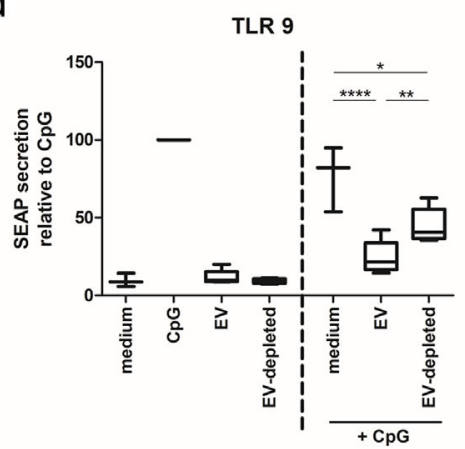

e

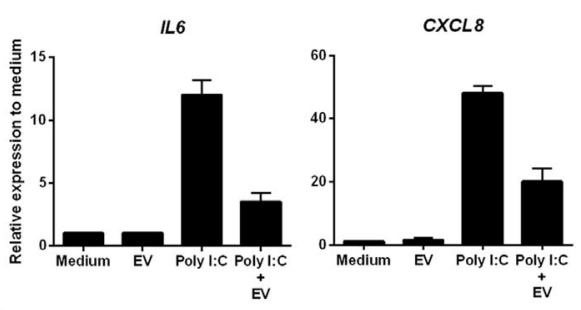

f

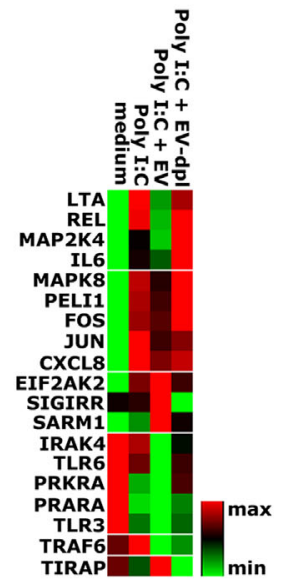

g

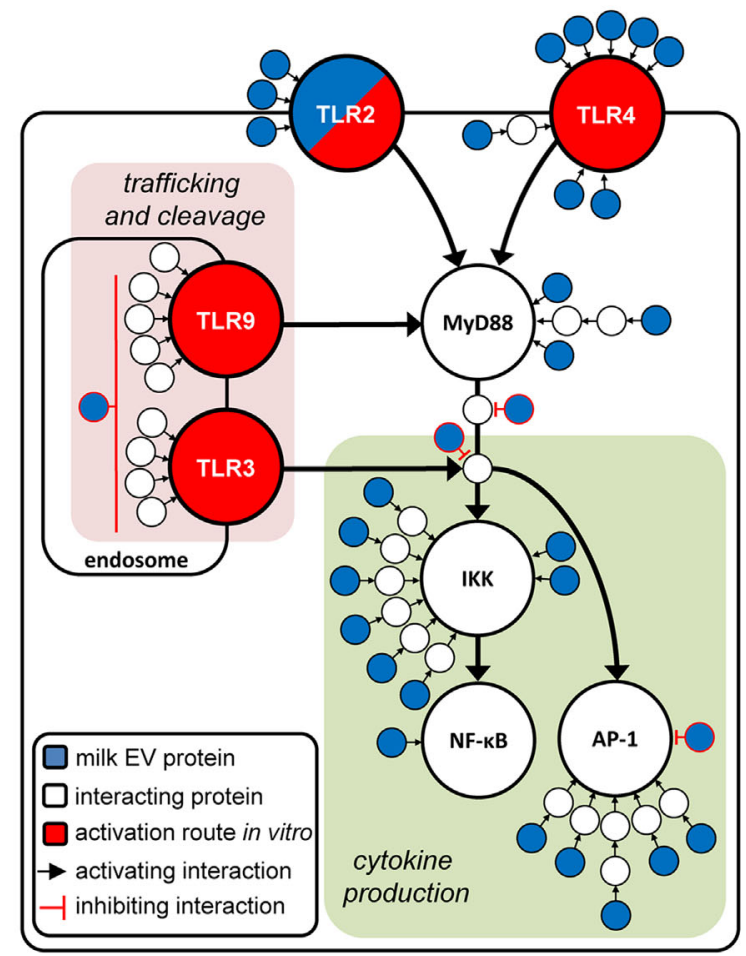

h

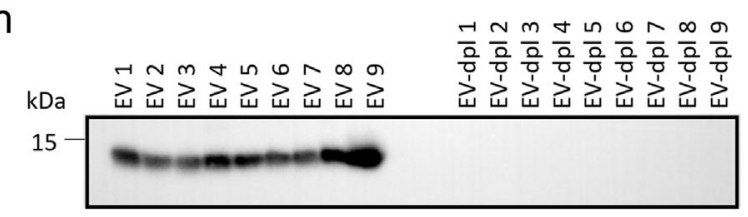

i

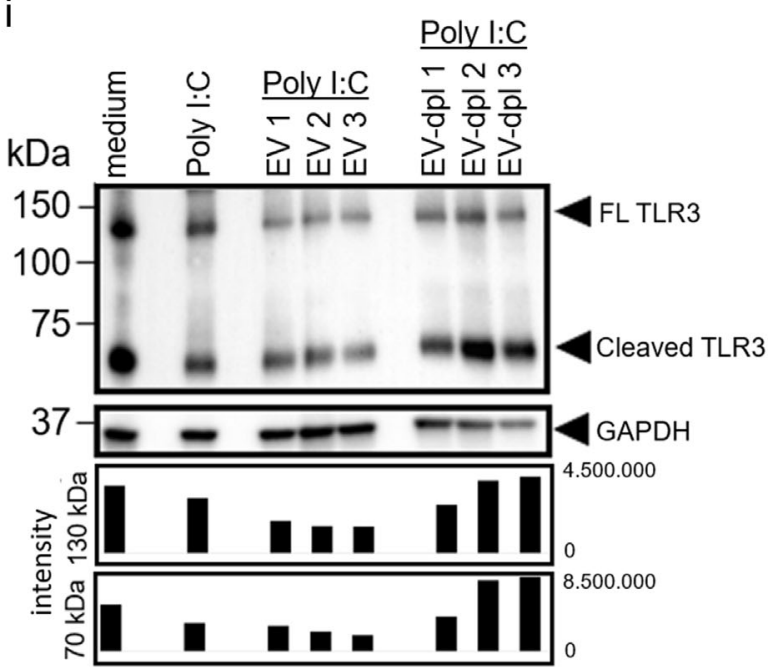

F I G URE 3 Human milk EVs inhibit agonist-induced activation of endosomal TLR3 and TLR9, but not of cell surface TLR2 and TLR4. [a-d) Secretion of SEAP reporter protein was determined for TLR reporter cell lines cultured in indicated conditions either or not in response to agonist (Pam3CSK for TLR2, LPS for TLR4, Poly I:C for TLR3, and CpG ODN2006 for TRL9), with the TLR-specific agonist set to $100 \%$. Box and whisker plots contain data from a single technical replicate of three independent experiments with a total of four different milk donors. e) Relative gene expression of IL6 and CXCL8 in Ca9-22 cells cultured for $5 \mathrm{~h}$ with medium, EV, Poly I:C or Poly I:C + EV. Delta Ct-values to ACTB and GAPDH were calculated and expressed relative to medium controls. EVs from two different milk donors were used and PCR reaction was performed twice on a single technical replicate. Results are summarized in bar graphs as mean \pm SD. f) Heatmap representing the cellular gene expression profile of Ca9-22 cells cultured for $4 \mathrm{~h}$ in the four different conditions (medium, agonist, EV + agonist, EV-depleted + agonist) in a gradient running from minimal gene expression (green) to maximal expression (red) for each gene analyzed (see Supplementary Figure 5 for complete dataset). Data is from 1 technical replicate derived from 1 experiment, with 1 milk donor. g) Schematic representation of key signaling pathways involved in TLR signaling of the individual TLRs tested which are shown in one Figure. TLR2, TLR4 and TLR9 signal via MyD88, while TLR3 signals via TRIF (not shown) to NF-kB (via IKK) and/or AP-1 leading to cytokine production. TLR2 and TLR4 are surface receptors, while TLR3 and TLR9 are sorted into the endosome where they are cleaved which enhances their signaling. Proteins from milk EVs are depicted in blue, while interacting cellular proteins are 
Liew et al., 2005). The enhancing effect of milk EVs was also observed for several genes that were downregulated by the agonist (IRAK4, TLR6, PRKRA, PPARA and TLR3). Finally, opposite effects of agonist-induced activation were observed in the absence or presence of milk EVs (TRAF6 and TIRAP) (Figure 3f). Collectively, the gene expression data indicate that milk EVs do not passively prevent TLR triggering by sequestering TLR ligands, but that they modulate TLR responses in an active manner. To assess which milk EV proteins could be involved in TLR modulation, we performed enrichment, network-, and functional annotation analysis and identified 26 significantly enriched GO-terms with $130 \mathrm{EV}$ proteins associated to TLR signalling, of which 110 proteins formed protein-protein networks (Supplementary File 3 and Supplementary Figure 6A).

From this analysis we extracted the key TLR signalling pathways targeted by milk EVs, leading to cytokine production or regulation of TLR trafficking and processing (Supplementary Figure 6B and Figure 3g). Besides the presence of low levels of endotoxin in the milk samples (Supplementary Figure 4), based on this model, the TLR2 and TLR4 activating effect of milk EVs in the absence of TLR agonists (Figure $3 \mathrm{a}$ and $3 \mathrm{~b}$ ) can be explained by the presence of several TLR2 and TLR4-stimulating proteins associated to milk EVs. As the signalling cascades for both plasma membrane and endosomal TLRs converge, no distinctive regulatory mechanism could be identified in our model that explains the inhibition of endosomal TLRs signalling. However, in contrast to TLR2 and TLR4, endosomal TLRs require specific trafficking and processing which involves transportation into the endosome followed by proteolysis via cathepsins, which enhances their signalling (Ewald et al., 2008; Garcia-Cattaneo et al., 2012; Gay et al., 2014). From our functional annotation analysis, we identified the cathepsin inhibitor cystatin-B (CSTB) (Turk et al., 1997), and verified its presence in milk EVs using immunoblotting (Figure 3h). Although CSTB has not been reported to be involved in the modulation of endosomal TLR cleavage, we explored whether milk EVs could affect the unprocessed full length and the cleaved form of TLR3 after agonist-induced activation of epithelial cells. Indeed, we observed less cleaved TLR3, however the abundance of full length TLR3 was also lower (Figure 3i). This suggests that milk EVs can modulate expression, or might interfere with TLR3 synthesis, for which the inhibition of TLR3 gene expression is indicative (Figure 3f). Taken together, our data show that milk EVs selectively inhibit agonist-induced endosomal TLR signalling, regulate downstream gene expression and reduce cellular full length and cleaved TLR3 expression. The selective regulation of key hotspots in TLR signalling could support the colonization of the mucosa in the new-born, as it has been shown that differential NF- $\kappa$ B activation is essential for immune homeostasis and tolerance to commensal bacteria (Chung \& Dale, 2008; Lee et al., 2006).

In contrast to innate immune cells, cells from the adaptive immune system are educated during postnatal development, whereby CD4+ T cells located in the MALT differentiate into T helper (Th) and regulatory subsets (Masopust \& Schenkel, 2013; Van Den Broek et al., 2018). Since epithelial cells can endocytose milk EVs (Liao et al., 2017) and selectively transport macromolecular structures from the apical to basolateral compartments via transcytosis (Tuma \& Hubbard, 2003), it is assumed that milk EVs can reach cells underlying the epithelial barrier. Therefore, we evaluated the effect of milk EVs on T cell activation and differentiation. When CD4+ T cells were activated in the presence of milk EVs, they were halted in their proliferation (Figure $4 \mathrm{a}$ and $4 \mathrm{~b}$ ). This inhibitory effect was dose-dependent (Figure $4 \mathrm{c}$ and Supplementary Figure 7 ) and transferrable to the EV-depleted control (Figure $4 \mathrm{~d}$ and Supplementary Figure 8). In order to determine whether milk EVs only interfered in proliferation and not differentiation, we monitored the ratio of naïve CD45RA + to memory CD45RO+ T cells (Sallusto et al., 2004; Van Den Broek et al., 2018). As expected, activation of $\mathrm{T}$ cells skewed the $\mathrm{T}$ cell population towards CD45RO at the expense of CD45RA (Figure 4e and 4f). In contrast, addition of milk EVs during $\mathrm{T}$ cell activation prevented this switch and $\mathrm{T}$ cells retained CD45RA expression (Figure 4e and 4f).This effect was dose-dependent (Supplementary Figure 9) and milk EVs were able to transfer this effect to the EV-depleted control in a dose-dependent manner (Supplementary Figure 10).The inhibitory effect of milk EVs was not merely via blocking proliferation of memory T cells (CD45RO), as proliferation of highly purified naïve CD4+CD45RA+ T cells was also inhibited (Supplementary Figure 11). This indicates that milk EVs inhibit activation of naïve $\mathrm{T}$ cells and their transition to a memory phenotype. Further evidence for impaired $\mathrm{T}$ cell activation in response to milk EVs was found by the inhibited release of a wide range of different Th subset-associated cytokines, for example, IFN- $\gamma$ (Th1), IL-5 (Th2), IL-9 (Th2, Th9 and Th17), IL-10 (Th2 and regulatory T cell: Treg), IL-13 (Th2), IL-17A and IL17F (Th17), and IL-22 (Th1, Th17) (Figure 4g). Suppression of proliferation and cytokine production could be indicative for Treg induction (Van Den Broek et al., 2018), and it has been previously reported that a crude preparation of human milk EVs induced expression of the Treg-associated transcription factor FoxP3 (Admyre et al., 2007). Yet, after exposure of activated purified T cells with a near physiological amount of milk EVs, we did not observe the induction of the typical Treg phenotype with high expression of CD25 and FoxP3 in the absence of CD127 (Supplementary Figure 12).

shown in white. Type of interactions between proteins is either shown as activating or inhibiting. In every assay, the individual TLR was activated via its specific ligand, which is shown in red. Note that TLR2 is present in milk EVs as well as in the TLR2 reporter cells. h) Western blot analysis for the presence of Cystatin-B (CSTB; expected size $11 \mathrm{kDa}$; exposure time $19 \mathrm{~s}$ ) in purified milk EVs or in EV-depleted control. Data is from one experiment with a single sample from nine different milk donors. i) Western blot analysis for the presence of TLR3 on whole cell lysate of Ca9-22 cells cultured in medium alone or stimulated with poly I:C with or without milk EVs or EV-depleted control. Full length (FL) TLR3 (expected size $130 \mathrm{kDa}$ ) and cleaved TLR3 (expected size $70 \mathrm{kDa}$ ) are visible. GAPDH (expected size $36 \mathrm{kDa}$ ) was used as a loading control and applied to normalize the intensity of the FL band and cleaved band (shown as volume intensity below the blot) in order to quantitatively compare signals. A total of three different milk donors were tested in a single experiment. Significance was calculated with 2-way mixed model analysis and Bonferroni correction and significance was defined as ${ }^{*} P<0.05$; ${ }^{* *} P<0.01$; ${ }^{* *} P<0.001$ and $\left.{ }^{\star * * *} P<0.0001\right]$ 
a

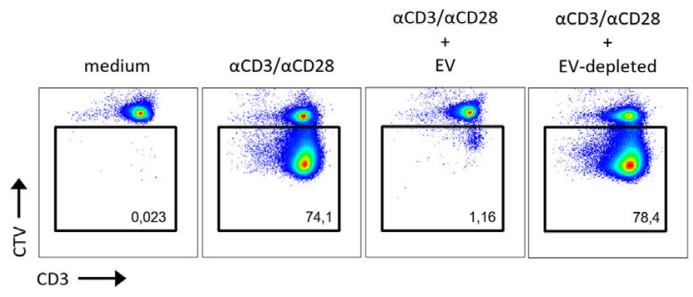

C

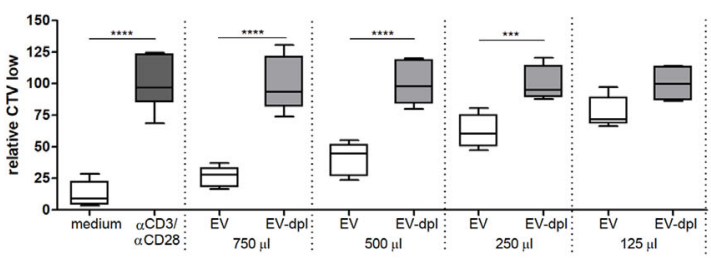

e

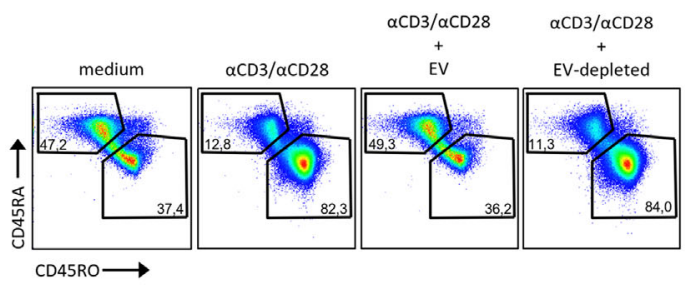

g
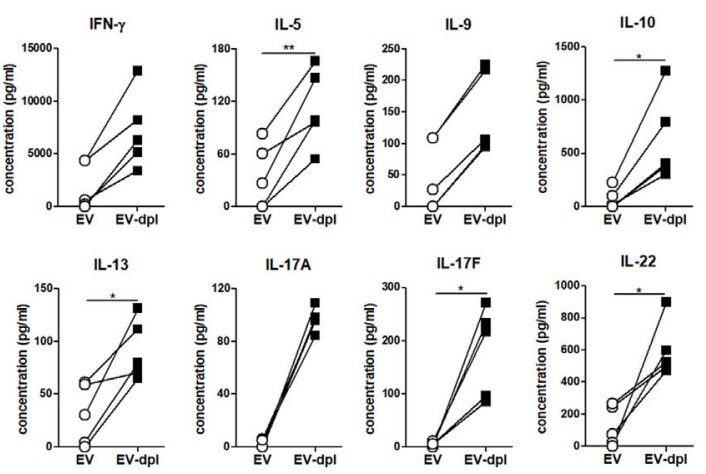

i

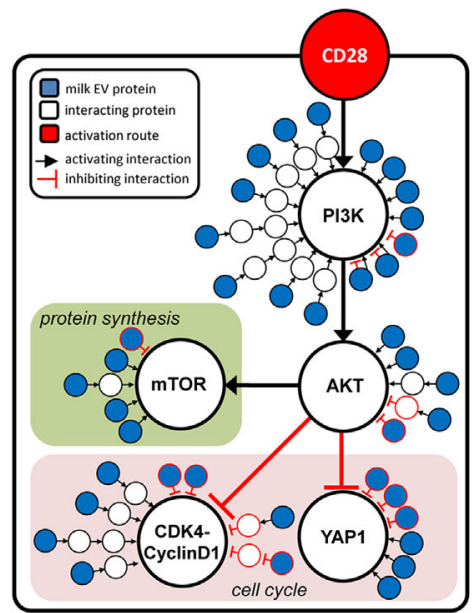

b

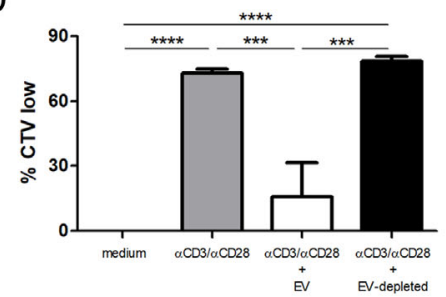

d
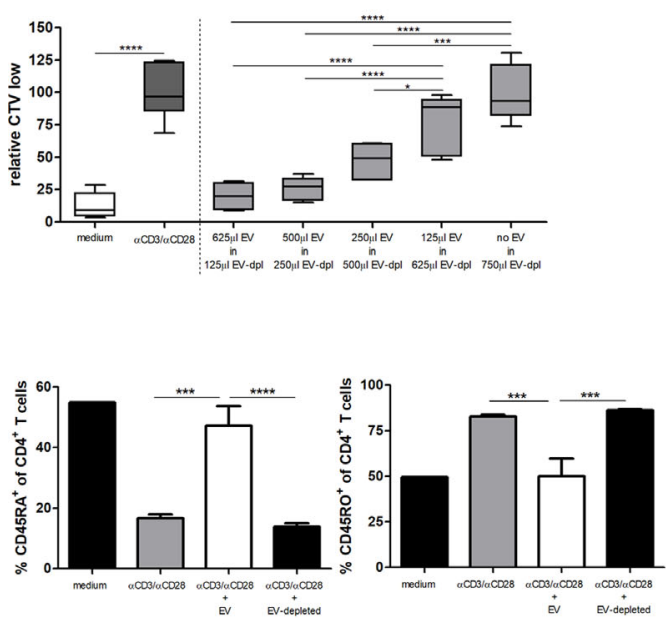

h
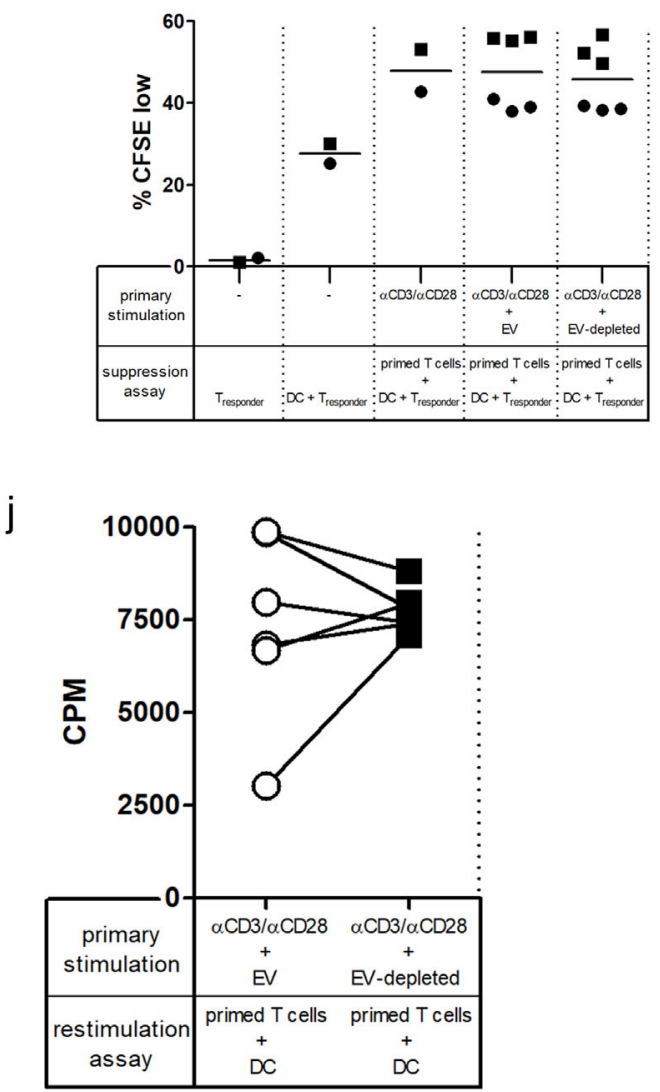

F I G URE 4 Human milk EVs transiently inhibit CD4+ T cell activation and retain cells in a naïve phenotype. [a-f) Purified CD4+ T cells were labelled with Cell Trace Violet (CTV) and incubated with medium, $\alpha \mathrm{CD} 3$ and $\alpha \mathrm{CD} 28(\alpha \mathrm{CD} 3 / \alpha \mathrm{CD} 28)$, or $\alpha \mathrm{CD} 3 / \alpha \mathrm{CD} 28$ in the presence of EV or EV-depleted control for 6 days. a) Representative dot plots of CTV dilution in response to the indicated conditions. Percentage CTV ${ }^{\text {low }}$ cells (gate) are expressed as a fraction of total CD4+ T cells. b) Quantification of the percentage CTV ${ }^{\text {low }} \mathrm{CD} 4+\mathrm{T}$ cells following incubation with the indicated conditions. Bars summarize mean \pm SD of a single technical replicate of a representative experiment of two independent experiments using different $\mathrm{T}$ cell donors $(\mathrm{n}=2)$ and different milk donors $(\mathrm{n}=2$ 
Additionally, we functionally tested the inhibitory capacity of T cells exposed to milk EVs. For this, CD4+ T cells were first stimulated in the presence of milk EVs, recovered and cultured with fresh CD4+ T-responder cells. As expected, in this suppression assay the milk EV-primed T cells were not suppressive (Figure $4 \mathrm{~h}$ ). To assess which pathways might be targeted by milk-EV proteins in the observed inhibition of T cell activation, we used enrichment analysis and identified a total of 48 significantly enriched GO-terms containing 165 proteins, of which 137 proteins were part of protein-protein interaction networks (Supplementary File 3 and Supplementary Figure 13A). Using functional annotation analysis, we could link the majority of these interacting proteins to relevant signalling cascades downstream of $\mathrm{CD} 28$, resulting in the inhibition of cell cycle and the stimulation of mTOR (Supplementary Figure 13B and Figure 4i). Although we do not provide direct molecular evidence, this model provides an explanation for the inhibition of proliferation and cytokine production by milk EVs, as the inhibition of downstream CD28 signalling can result in the retained naïve CD4+ T cell phenotype (Harding et al., 1992). Furthermore, the absence of Treg induction by milk EVs becomes evident, as mTOR and PI3K inhibit FoxP3 expression in T cells (Merkenschlager \& Von Boehmer, 2010). These findings suggest that milk EVs directly inhibit CD4+ T cell activation, without $\mathrm{T}$ cell tolerance or Treg induction. To test this, we activated naïve $\mathrm{CD} 4+\mathrm{T}$ cells in the presence of milk EVs, recovered the cells and restimulated them with allogeneic DC. Indeed, T cells that were primed in the presence of milk EVs, proliferated after restimulation, to the same extent as restimulated T cells primed with EV-depleted control (Figure 4j). This demonstrates that the presence of milk EVs causes a transient inhibition of $\mathrm{T}$ cell activation which is reversible. Based on our findings we propose that milk EVs create a temporary increased threshold for $\mathrm{CD} 4+\mathrm{T}$ cell activation. Raising this threshold might help the infant to cope with the high antigenic load to which it is exposed after birth, creating tolerogenic conditions that are optimal for development (Basha et al., 2014; Caballero \& Pamer, 2015).

Although human milk is known to aid the postnatal development and maturation of the GI tract, and maintenance of immune homeostasis (Cummins, 2002; He et al., 2016; Renz et al., 2011; Turfkruyer \& Verhasselt, 2015), the exact effects on the oral mucosa has remained understudied. Here we demonstrate by using in vitro model systems that purified EVs from human milk can promote gingival cell re-epithelialization, modulate epithelial endosomal TLR responses and transiently control T cell activation (Supplementary Figure 14). As EVs are heterogeneous in composition, the net-effect on the target cell will be determined by the collective EV cargo delivered and relevant pathways targeted. Therefore, EV-induced effects result from the concerted action of numerous biomolecules delivered, rather than from a single regulatory molecule. This is underscored by our models derived from the functional annotation analysis of the milk EV proteome, in which potential interactions of protein cargo occurs throughout the signalling cascades and where nodes of high interaction affect key signalling proteins. Whereas some 'regulatory hotspots' like PI3K, are prominent in all three biological processes studied, other unique hotspots were identified in each biological assay. In the end, these models do not establish a causal link and therefore molecular or functional validation remains essential to understand how milk EV cargo targets cellular components. Besides proteins, milk EV contain other cargo like miRNAs, from which several miRNAs (let-7 family members let-7a, let-7b, let-7f, and miR-148a) have validated target genes that are involved in signal transduction and cell growth and/or maintenance (Herwijnen et al., 2018). Therefore, milk EVs have the potential to not only regulate cellular processes on a protein level via their protein cargo, but can also do this on a transcriptional level for

and 3). $\mathrm{c}$ and d) Relative expression of CTVlow CD4+ T cells following incubation with the indicated conditions, with each paired control set to $100 \%$. Box and whisker plots contain data of a single experiment using $n=1 \mathrm{~T}$ cell donor and $\mathrm{n}=3$ different milk donors. e) Representative dot plots of CD4+ T cells stained for CD45RA and CD45RO after culture in the indicated conditions. Percentage of CD45RA+/CD45RO- and CD45RO+/CD45RA- cells in gates are expressed as a fraction of total CD4+ T cells. f) Quantification of the percentage CD45RA+ and CD45RO+ cells of total CD4+T cells following incubation with the indicated conditions. Bars summarize mean \pm SD of a single technical replicate of a representative experiment of two independent experiments using different T cell donors $(n=2)$ and different milk donors $(n=2$ and 3$)$. g) Cytokine profiles of stimulated CD4+ T cells incubated with EV or EV-deleted controls. The following values were obtained for $\alpha \mathrm{CD} 3 / \alpha \mathrm{CD} 28$ stimulated controls (not shown in the bar graph), provided as mean \pm SD: IFN- $\gamma: 4514 \pm 1757 \mathrm{pg} / \mathrm{ml}$, IL-5: 89 \pm 30 pg/ml, IL-9: $123 \pm 37$ pg/ml, IL-10: $330 \pm 243$ pg/ml, IL-13: $99 \pm 22$ pg/ml, IL-17A: $13 \pm 8$ pg/ml (compared to $97 \pm 8$ pg/ml with EV-depleted), IL-17F: 68 $\pm 47 \mathrm{pg} / \mathrm{ml}$ (compared to $180 \pm 76 \mathrm{pg} / \mathrm{ml}$ with EV-depleted), IL-22: $346 \pm 59 \mathrm{pg} / \mathrm{ml}$. Data is shown for day 6 as highest secretion was seen for $\alpha \mathrm{CD} 3 / \alpha \mathrm{CD} 28$ controls on this day. Graphs summarize results of a single technical replicate from two independent different $\mathrm{T}$ cell donors $(\mathrm{n}=2)$ and different milk donors $\left(\mathrm{n}=2\right.$ and 3). h) Suppression assay performed with CFSE-labelled CD4+ $\mathrm{T}_{\text {responder }}$ cells incubated with allogeneic monocyte-derived DC and autologous CD4+ $\mathrm{T}$ cells that received primary stimulation with $\alpha \mathrm{CD} 3 / \alpha \mathrm{CD} 28$ or with $\alpha \mathrm{CD} 3 / \alpha \mathrm{CD} 28$ in the presence of EVs or the EV-depleted control. After priming, the cells were washed, irradiated and added to an allogeneic mixed lymphocyte reaction (MLR). Plotted are single points for the average of technical triplicates, shown as a percentage of total $\mathrm{T}_{\text {responder }}$ cells. Data is representative of a single technical replicate from two independent experiments (with two individual donors) using three different milk donors. i) Schematic representation of key signaling pathways involved in regulating $\mathrm{T}$ cell activation: downstream signaling of CD28 with inhibition of in cell cycle (e.g., YAP1 and CDK4-CyclinD1 via PI3K-AKT) and activation of mTOR (via PI3K-AKT). Proteins from milk EVs are depicted in blue, while interacting cellular proteins are shown in white. Type of interactions between proteins is either shown as activating or inhibiting. CD28 is highlighted in red, as this receptor was stimulated. j) Restimulation assay with purified CD45RA+CD4+ T cells $(n=2)$ that received a primary stimulation with $\alpha \mathrm{CD} 3 / \alpha \mathrm{CD} 28$ in the presence of EVs or EV-depleted control $(n=3)$. Cells were subsequently restimulated with allogeneic monocyte-derived DC in the absence of EV and EV-depleted controls. Restimulation was performed in triplicate and the average values plotted as a single point. Proliferation was measured by ${ }^{3} \mathrm{H}$-thymidine incorporation and data is expressed as counts per minute (CPM). Significance was calculated with one-way ANOVA and Tukey's or Sidak's multiple comparisons test (b, $\mathrm{c}$ and d; for $\mathrm{f}$ medium controls were not normally distributed and were therefore omitted from statistical analysis), paired $t$-test, or Wilcoxon matched pairs signed rank test (g and j), or Kruskal-Wallis and Dunn's multiple comparison test (h) and significance was defined as ${ }^{*} P<0.05$; $^{* *} P<0.01$; ${ }^{* *} P<0.001$ and $\left.{ }^{* * * *} P<0.0001\right]$ 
instance via their miRNAs. In that respect we postulate that for the biological function of EVs, heterogeneity of the EV population and their respective cargo ensures fine-tuning with respect to specific cellular response. Future studies are needed to validate our in vitro findings in vivo and to determine which $\mathrm{EV}$ subsets interact with specific recipient cells, as it is likely that EV subsets will contain specific cargos that are geared towards distinct functions in target cells, and that their composition is controlled by environmental cues from the mother (Torregrosa Paredes et al., 2014).

In summary, EVs are multifactorial and bioactive components of human milk that can interact with various cell types found in the oral mucosa, creating a window of opportunity for regulated development of the epithelial barrier and the innate and adaptive immune system of the new-born.

\section{1 | METHODS}

\subsection{Human milk collection}

Human milk was collected as previously described (Zonneveld et al., 2014). Briefly, fresh and mature milk samples were collected by means of an electric breast pump by 16 healthy mothers, with a mean age of $33 \pm 2.4$ years who were at a lactational stage of 3 to 15 months (with an average of $7.2 \pm 3$ months). The milk was prevented from cooling down and EV isolation started within $30 \mathrm{~min}$ after collection. Informed consent was signed by all donors and this study was approved by the local ethics committee.

\subsection{Human milk EV isolation and EV-depleted milk control isolation}

Isolation of milk EVs was performed as previously described with some modifications for functional analysis (Zonneveld et al., 2014). Whole milk was centrifuged at $22^{\circ} \mathrm{C}$ for $10 \mathrm{~min}$ at $3000 \mathrm{~g}$ (Beckman Coulter Allegra X-12R, Fullerton, CA, USA). After removal of the cream layer the harvested milk supernatant was centrifuged at $3000 \mathrm{~g}$ again at $22^{\circ} \mathrm{C}$ and stored at $-80^{\circ} \mathrm{C}$ until further processing. Thawed supernatant was centrifuged in polyallomer SW40 tubes (Beckman Coulter) at $5000 \mathrm{~g}$ for $30 \mathrm{~min}$ at $4^{\circ} \mathrm{C}$ and subsequently at 10,000 $\mathrm{g}$ (Beckman Coulter Optima L-90K with a SW40Ti rotor). Next, $6.5 \mathrm{ml}$ aliquots of the 10,000 g supernatant were loaded on top of a 60\%-10\% iodixanol gradient (Optiprep, Progen Biotechnik GmbH, Heidelberg, Germany) in a SW40 tube. Gradients were ultracentrifuged at 192,000 g (Beckman Coulter Optima L-90K with a SW40Ti rotor) for 15-18 h. Following this centrifugation, the resulting EV-depleted milk supernatant $(6.5 \mathrm{ml})$ left on top of the gradient was collected and loaded onto a new iodixanol gradient and ultracentrifuged once more and further processed identically to milk EV samples, to obtain a donor-matched procedural milk control. The EV and EV-depleted samples from the iodixanol gradients were collected in fractions of $500 \mu \mathrm{l}$ and densities of 1.06-1.19 $\mathrm{g} / \mathrm{ml}$ were pooled, as these had a high expression of the EV-associated marker CD9 in Western blot for the EV sample (data not shown). Iodixanol was removed from samples by size exclusion gel filtration using a $20 \mathrm{ml}$ column (Bio-Rad Laboratories, Hercules, CA, USA) packed with $15 \mathrm{ml}$ Sephadex $g 100$ (Sigma-Aldrich, St. Louis, MO, USA) and elutriating 24 fractions of $1 \mathrm{ml}$ with phenol red free RPMI 1640, or DMEM medium (Gibco, Invitrogen, Carlsbad, CA, USA). Eluates 3-9 contained EVs as these had a high protein concentration and a high expression of the EV-associated marker CD63 (data not shown). The EV-containing eluates were pooled and supplemented with $10 \%$ heat-inactivated foetal calf serum (FCS; Sigma-Aldrich), or 0.1\% BSA (Sigma-Aldrich), $2 \mathrm{mM}$ ultraglutamine (BioWhittaker, Lonza, Switzerland), and $100 \mathrm{IU} / \mathrm{ml}$ penicillin and $100 \mathrm{mg} / \mathrm{ml}$ streptomycin (Gibco). Samples were frozen at $-80^{\circ} \mathrm{C}$ until use. The isolated milk EVs were characterized by Western blot for EV-associated markers CD9, CD63, Flotillin-1 and HSP70, as well as the non-EV marker lactoferrin. Concentrations of EVs used for in vitro assays were within or below the physiological range, as the starting volume of the $10,000 \mathrm{~g}$ milk supernatant $(6.5 \mathrm{ml})$ from which the milk EVs were isolated is in the range of the pooled eluates $(7 \mathrm{ml})$. We have submitted all relevant data of our experiments to the EV-TRACK knowledgebase (EV-TRACK ID: EV200007) (Van Deun et al., 2017).

\subsection{Nanoparticle tracking analysis}

NTA measurements were performed with a NanoSight NS300 (Malvern Instruments Ltd, UK) equipped with a $405 \mathrm{~nm}$ laser, a high sensitivity sCMOS camera and a NanoSight Syringe Pump (Malvern Instruments Ltd, UK) for flow mode measurements. The NTA 3.2 software build 3.2.16 was used for data acquisition and processing. Each sample was diluted in low endotoxin PBS (1:100 for milk EVs, $1: 10 \mathrm{EV}$-depleted controls) to obtain a concentration in the range of $1^{\star} 10^{8}-1^{\star} 10^{9}$ particles $/ \mathrm{ml}$ and particles in frame between 70 and 110. For each sample 3 captures of $60 \mathrm{~s}$ each were acquired with a syringe pump flow rate of $20 \mathrm{AU}$ and temperature controlled at $20^{\circ} \mathrm{C}$. Captures were obtained with camera level 13 and default combination of shutter and gain. Capture processing was performed with detection threshold 9 , viscosity set to water $(0,9 \mathrm{cP})$ and automatic blur and minimum 
track length. The number of valid tracks in EV samples was always approximately 3 times greater than the recommended limit of 1000 (Gardiner et al., 2013).

\section{4 | SDS page and Western blotting}

For characterization of samples during EV isolation, $100 \mu \mathrm{l}$ aliquots were collected from iodixanol gradient Optiprep fractions, or eluates from the size exclusion gel filtration column. Isolated milk EVs, EV-depleted sample, or fractions were pelleted by centrifugation for 65 min at 100,000 g (in a Beckman Coulter Optima Max-XP with a TLA-55 rotor) in polyallomer microcentrifuge tubes (Beckman). Pelleted fractions were resuspended in sample buffer (62.5 mM Tris pH 6.8, 2\% SDS, 10\% Glycerol), while pelleted EVs or EV-depleted sample was resuspended in RIPA buffer (40 mM Tris pH 8.0, $150 \mathrm{mM} \mathrm{NaCl}, 1 \%$ Triton $\mathrm{X}-100,0.5 \%$ Na-deoxycholate, $0.1 \%$ SDS). Whole cell lysates from Ca9-22 cells were prepared from $5.2^{\star} 10^{5}$ cells in $30 \mu \mathrm{l}$ in RIPA buffer. Protein content of whole cell lysate was measured with the BCA protein assay (Pierce, Thermo Scientific, Landsmeer, Netherlands) to quantify the protein content of the samples in order to equalize for input material for SDS page. Samples were heated at $95^{\circ} \mathrm{C}$ for $3 \mathrm{~min}$ and run on an $8 \%-16 \%$ or $4 \%-20 \%$ TGX-Criterion gel (Bio-Rad). The separated proteins were transferred to PVDF membranes and blocked in PBS containing 0.2\% fish skin gelatin (Sigma-Aldrich) and 0.1\% Tween-20. Proteins were detected by immunoblotting using mouse anti-human CD9 (clone HI9a, BioLegend, dilution 1:1000), mouse anti-human CD63 (clone TS63, Abcam, dilution 1:1000), mouse anti-human flotillin-1 (clone 18, BD Biosciences, MA, USA, dilution 1:500, sample reduced with $\beta$-mercaptoethanol) and mouse anti-human HSC70/HSP70 (clone N27F3-4, ENZO dilution 1:1000, sample reduced with $\beta$-mercaptoethanol) and rabbit anti-human lactoferrin (polyclonal, Abcam, dilution 1:5000), rabbit anti-human TLR3 (D10F10, Cell Signaling TECHNOLOGY, dilution 1:1000, sample reduced with $\beta$-mercaptoethanol), mouse anti-human GAPDH (mAbcam 4984, Abcam, dilution 1:1000), mouse anti-human CSTB (clone 225228, R\&D Systems, dilution 1:500, sample reduced with $\beta$-mercaptoethanol). Goat anti-mouse-HRP (Jackson Immuno Research, Suffolk, UK; dilution 1:10,000), or goat anti-rabbit-HRP (DAKO, dilution 1:1000) was used as secondary antibody. HRP conjugated antibodies were detected using SuperSignal West Dura Chemiluminescent Substrate (Thermo Scientific and ChemiDoc XRS and Image Lab 5.1 (Bio-Rad).

\section{5 | Re-epithelialization assay}

Ca9-22 (JCRB0625) gingival epithelial cells (JCRB Cell Bank, Osaka, JP) were cultured in DMEM containing Glutamax (Gibco) and supplemented with 10\% FCS (Gibco), $100 \mathrm{U} / \mathrm{ml}$ penicillin and $100 \mu \mathrm{g} / \mathrm{ml}$ streptomycin (Sigma-Aldrich). The reepithelialization assay was performed as previously described (Fernandez-Gutierrez et al., 2017). For this, cells were seeded at $3.5 \times 10^{4}$ cells/well in 96-well flat bottom tissue culture treated plates (BD Falcon, Corning, NY, USA) and left to reattach and form a confluent monolayer overnight. Cells were starved in FCS-free DMEM for $2 \mathrm{~h}$ prior to experiments and labelled during the last 20 min of starvation with $2 \mu \mathrm{M}$ CellTracker Red CMTPX and $2 \mu \mathrm{g} / \mathrm{ml}$ Hoechst 33342 (both from Molecular Probes, Eugene, OR, USA). A gap was made in the cell monolayers of every well using an HTS Scratcher (Peira, Antwerp, BE). Cells were washed twice with PBS (Gibco) and cultured in FCS-free DMEM as non-treated control, $4 \mathrm{ng} / \mathrm{ml}$ human transforming growth factor $\alpha$ (TGF $\alpha$; R\&D Systems, MN, USA) as a positive control, or with $100 \mu \mathrm{l}$ milk EVs, or $100 \mu \mathrm{l} \mathrm{EV-depleted} \mathrm{control.} \mathrm{For} \mathrm{inhibition}$ of migration via $\mathrm{p} 38 \mathrm{MAPK}$ or proliferation via MEK1/2 validated and selective inhibitors were used, respectively $10 \mu \mathrm{M}$ of p38 inhibitor SB203580 (4-(4-fluorophenyl)-2-(4-methylsulfinylphenyl)-5-(4-pyridyl)-imidazole (Cuenda et al., 1995; Kumar et al., 1999)) or $10 \mu \mathrm{M}$ of MEK1/2 inhibitor U0126 (Favata et al., 1998) (both from Cell Signaling Technology, Danvers, MA, USA). Importantly, the concentrations used were in a range that would not induce off-target side effects (Favata et al., 1998; Kumar et al., 1999). Cells were monitored by live cell imaging using the BD Pathway 855 Bioimaging System (BD Biosciences). Images of the same frame of each well were acquired every $20 \mathrm{~min}$ for $5 \mathrm{~h}$ or until the gaps in the positive controls were closed. Image segmentation and data analysis were carried out using CellProfiler 2.1.1 (https://www.cellprofiler.org/) and FCS Express 4 Plus (De Novo Software, Glendale, CA, USA). A nonlinear least squares regression was used to fit the modified Gompertz function (Fernandez-Gutierrez et al., 2017) through the re-epithelialization measurements obtained per well. The repair rate parameter derived from the model was used to calculate the average re-epithelialization rate (cells/min) from technical triplicates. An integrated pipeline for image analysis, single cell enumeration, and kinetic modelling is available online through the Galaxy platform (Fernandez-Gutierrez et al., 2018) and a detailed protocol of the assay can be found in Nature Protocol Exchange (FernandezGutierrez et al., 2020). To determine cell displacement, sequential images per treatment were loaded into Fiji (ImageJ), stacked ( $z$ as time), and converted into a hyperstack. The stacks were subsequently tracked using TrackMate (Tinevez et al., 2017) (v. 5.2.0). Cell nuclei were detected using LoG detector (estimated blob diameter 0.115 , threshold 15 , with sub-pixel localization). All selected spots were used for further analysis (no thresholding) and spots were tracked using the linear motion LAP tracker (initial search radius 0.2 , search radius 0.3 , max frame gap 2 ). The tracks were pseudo-coloured according to the total track displacement that was scaled for each individual image series. Feature tables for time and individual tracks as well as their corresponding cell 
displacement were exported. Subsequently, the tracks were visualized by capture overlay (HyperstackDisplayer) with their corresponding colours for total track displacement.

\subsection{TLR reporter assay}

HEK-Blue-hTLR2, HEK-Blue-hTLR3, HEK-Blue-hTLR4, and HEK-Blue-hTLR9 reporter cell lines (Invivogen, Toulouse, FR) were cultured in DMEM medium containing Glutamax (Gibco), supplemented with 8.5\% heat-inactivated FCS (Bodinco), 50 units $/ \mathrm{ml}$ penicillin (Sigma-Aldrich), $50 \mu \mathrm{g} / \mathrm{ml}$ streptomycin (Sigma-Aldrich) and $100 \mu \mathrm{g} / \mathrm{ml} \mathrm{Normocin} \mathrm{(Invivogen).} \mathrm{Addi-}$ tionally, the HEK-Blue hTLR9 culture medium was supplemented with $10 \mu \mathrm{g} / \mathrm{ml}$ blasticidin (Invivogen) and $100 \mu \mathrm{g} / \mathrm{ml}$ zeocin (Invivogen), the HEK-Blue hTLR3 culture medium with $30 \mu \mathrm{g} / \mathrm{ml}$ blasticidin and $100 \mu \mathrm{g} / \mathrm{ml}$ zeocin and the HEK-Blue hTLR4 and -hTLR2 culture medium with 1x HEk-Blue Selection (Invivogen). For experiments, cells were cultured in 96-well flat bottom tissue culture treated plates with $90 \mu \mathrm{l}$ milk EVs or EV-depleted with a final volume/well $110 \mu \mathrm{l}$. Agonists used were $100 \mathrm{ng} / \mathrm{ml}$ Pam3CSK, $5 \mu \mathrm{g} / \mathrm{ml}$ Poly I:C, $10 \mathrm{ng} / \mathrm{ml}$ LPS-EK, or $1 \mu \mathrm{g} / \mathrm{ml} \mathrm{CpG} \mathrm{ODN2006} \mathrm{(all} \mathrm{from} \mathrm{Invivogen).} \mathrm{Cells} \mathrm{were} \mathrm{cultured}$ for $16 \mathrm{~h}$ after which supernatant was harvested and SEAP reporter protein secretion was determined using QUANTI-Blue detection medium (Invivogen) and measuring absorption at $650 \mathrm{~nm}$ on a 550 Microplate reader (Bio-Rad) as per manufacturer's instructions.

\section{7 | TLR stimulation of Ca9-22 cells}

Ca9-22 cells were seeded at $3.5 \times 10^{4}$ cells/well in 96-well flat bottom plates (Corning) with DMEM medium containing Glutamax (Gibco) and supplemented with 10\% FCS (Gibco) (Sigma-Aldrich), $100 \mathrm{U} / \mathrm{ml}$ penicillin and $100 \mu \mathrm{g} / \mathrm{ml}$ streptomycin (SigmaAldrich). The next day, the medium was replaced by FCS-free medium containing 0.1\% BSA (Sigma-Aldrich) and cells were stimulated with $100 \mu \mathrm{l}$ milk EVs or EV-depleted controls in the presence or absence of $5 \mu \mathrm{g} / \mathrm{ml}$ Poly I:C (Invivogen). After 4 or $5 \mathrm{~h}$, cells were washed with PBS (Gibco), harvested and stored at $-80^{\circ} \mathrm{C}$ in RLT buffer with $\beta$-mercaptoethanol from the RNeasy Mini prep Kit (Qiagen, Hilden, Germany) according to manufacturer's instructions until cDNA synthesis for RT ${ }^{2}$ Profiler PCR array. For TLR3 and GAPDH Western blot, cells were stored in RIPA buffer (40 mM Tris pH 8.0, $150 \mathrm{mM} \mathrm{NaCl,} \mathrm{1 \%} \mathrm{Triton} \mathrm{X-100,}$ $0.5 \%$ Na-deoxycholate, $0.1 \%$ SDS composition) buffer until use.

\section{8 | Endotoxin quantitation}

The gram-negative bacterial endotoxin concentration in the EV and EV-depleted samples was measured using the Pierce LAL Chromogenic Endotoxin Quantitation Kit (ThermoFisher, MA, USA) according to the manufacturer's instructions.

\section{$1.9 \quad$ | RT-qPCR and RT2 ${ }^{2}$ Profiler PCR array}

Total RNA was extracted from Ca9-22 cells after 4 or $5 \mathrm{~h}$ of culture using the RNeasy Mini prep Kit and cDNA was prepared using the RT ${ }^{2}$ First Strand Kit (Qiagen) or the qScript cDNA Synthesis Kit (Quanta Biosciences) according to the manufacturer's instructions. Real-time quantitative PCR (RT-qPCR) was performed on a Rotor-Gene Q2plex real-time cycler (Qiagen). For relative gene expressions of IL6 and CXCL8, delta Ct-values were log-transformed with ACTB and GAPDH as internal controls. For gene expression profiling, cDNA was added to the Human Toll-like receptor signalling pathway RT $^{2}$ Profiler PCR array (Qiagen) and run on an iCycler MyiQ (Bio-Rad). The relative expression levels of each gene were normalized to the expression level of 5 reference genes included in the array (ACTB, B2M, GAPDH, HPRT1 and RPLP0). Delta Ct-values were log-transformed and analyzed by the web-based software GeneGlobe Data Analysis Center (Qiagen). Genes for which gene expression levels had a Ct $>35$ in all test conditions were excluded from analysis.

\subsection{0 | CD4+ T cell isolation}

Human peripheral blood mononuclear cells (PBMC) were isolated from Buffy coats by Lymphoprep density gradient centrifugation (Axis-shield, Dundee, United Kingdom and Nycomed, Zurich, Switzerland). PBMC were cultured in RPMI 1640 supplemented with Glutamax and sodium pyruvate (Gibco), 2.5\% FCS and $100 \mathrm{IU} / \mathrm{ml}$ penicillin and $100 \mathrm{mg} / \mathrm{ml}$ streptomycin (Gibco). CD4+ T cells were isolated from PBMC using CD4+ T cell isolation kit (Miltenyi Biotec, Bergisch Gladbach, Germany) according to manufacturer's instructions (average purity of 95\%) and were used directly in experiments or fractionated into CD45RA+ and 
CD45RO+ subsets using anti-CD45RA-PE (UCHL-1, Dako) and anti-PE magnetic beads (Miltenyi Biotec) resulting in > 97\% pure T cell subpopulations.

\subsection{1 | T cell stimulation}

PBMCs were seeded at $4 \times 10^{6}$ cells $/ \mathrm{ml}$ in 12 wells plates (Corning) coated with $1.5 \mu \mathrm{g} / \mathrm{ml} \alpha \mathrm{CD} 3$ (CLB-T3/4.E, $1 \mathrm{XE}$ from Sanquin, Amsterdam, The Netherlands) and cultured in RPMI 1640 medium (Gibco) with 10\% FCS (Sigma-Aldrich), $750 \mu l$ milk EVs or EV-depleted controls in a total volume of $1 \mathrm{ml}$ and cultured for 6 days. Purified CD4+ T cells were seeded at $0.5 \times 10^{6}$ cells $/ \mathrm{ml}$ in 48 wells plates (Corning) coated with $0.5-1.5 \mu \mathrm{g} / \mathrm{ml} \alpha \mathrm{CD} 3$ (CLB-T3/4.E, 1XE from Sanquin, Amsterdam, The Netherlands) and cultured with $70 \mathrm{ng} / \mathrm{ml}-1 \mu \mathrm{g} / \mathrm{ml}$ soluble $\alpha$ CD28 (CLB-CD28/1, 15E8 from Sanquin), RPMI 1640 medium (Gibco) with 10\% FCS (Sigma-Aldrich), $750 \mu \mathrm{l} \mathrm{milk} \mathrm{EVs} \mathrm{or} \mathrm{EV-depleted} \mathrm{controls} \mathrm{in} \mathrm{a} \mathrm{total} \mathrm{volume} \mathrm{of} 1 \mathrm{ml}$ and cultured for the indicated amount of time. Notably, the final concentration of milk EVs was $75 \%$ of the physiological concentration of the EV preparations as obtained after SEC when $750 \mu \mathrm{l}$ of milk EVs were added. Hence, formally T cell modulation studies were performed with a sub physiological concentration. Additionally, lower volumes of milk EV samples were added to determine dose-dependent effects and add back experiments were performed in which milk EVs were added to the EV-depleted milk control in order to recover EV-mediated effects.

\subsection{Multiplex cytokine analysis}

Supernatants of stimulated T cells were harvested on day 6 of culture and analyzed using the LEGENDplex human Thelper 1/2/9/17 multiplex kit (BioLegend). Beads were acquired on a BD Canto II (BD Biosciences) and analyzed with LEGENDplex V7.0.

\subsection{3 | T cell suppression assay}

$\mathrm{T}$ cell suppression assay was performed as described previously (Van Der Aar et al., 2011). In brief, $\mathrm{CD} 4^{+} \mathrm{CD} 45 \mathrm{RA}{ }^{+} \mathrm{T}$ cells were activated for 5-6 days with $1.5 \mu \mathrm{g} / \mathrm{ml}$ plate bound $\alpha \mathrm{CD} 3$ (Sanquin) and $1 \mu \mathrm{g} / \mathrm{ml}$ soluble $\alpha \mathrm{CD} 28$ (Sanquin) in medium, or in the presence of EV or EV-depleted controls. T cells were subsequently harvested, irradiated (3,000 rad), washed and counted. Cells were replated at a 2:1 ratio with $0.5 \mu \mathrm{M}$ CFSE (Sigma-Aldrich) labelled autologous $\mathrm{CD} 4^{+} \mathrm{CD} 45 \mathrm{RO}^{+}$responder cells in the presence of monocyte-derived allogeneic DC. Cells were incubated for 5-6 days and CFSE dilution of T cells was determined on a BD Canto II (BD Biosciences).

\subsection{4 | Restimulation assay}

$10 \times 10^{6}$ cells $/ \mathrm{ml} \mathrm{CD}^{+} \mathrm{CD} 4 \mathrm{RA}^{+} \mathrm{T}$ cells were cultured for 3 days in the presence of $1.5 \mu \mathrm{g} / \mathrm{ml}$ plate bound $\alpha \mathrm{CD} 3$ (CLB-T3/4.E, 1XE Sanquin) and $1 \mu \mathrm{g} / \mathrm{ml}$ soluble $\alpha \mathrm{CD} 28$ (CLB-CD28/1, 15E8 Sanquin) in the presence or absence of milk EVs or EV depleted control. T cells were subsequently harvested, washed, counted and reseeded at a 4:1 ratio with allogeneic monocyte-derived DC generated as previously described (De Jong et al., 2002) in a mixed lymphocyte response. Cells were incubated for 3 days total and to determine $\mathrm{T}$ cell proliferation $11 \mathrm{KBQ} /$ well $^{3} \mathrm{H}$-thymidine (Radiochemical Center, Amersham, Little Chalfont, UK) was added. The incorporated ${ }^{3} \mathrm{H}$-thymidine was measured after $16 \mathrm{~h}$ by liquid scintillation spectroscopy.

\subsection{5 | Flow cytometry}

Expression of E-cadherin and EPCAM was determined after Ca9-22 gingival epithelial cells were cultured in DMEM containing Glutamax (Gibco), $100 \mathrm{U} / \mathrm{ml}$ penicillin and $100 \mu \mathrm{g} / \mathrm{ml}$ streptomycin (Sigma-Aldrich). Cells were seeded at $3.5 \times 10^{4}$ cells/well in 96-well flat bottom tissue culture treated plates (BD Falcon) and left to reattach and form a confluent monolayer overnight. Cells were starved in FCS-free DMEM for $2 \mathrm{~h}$ prior to experiments after which they were washed twice with PBS (Gibco) and cultured in FCS-free DMEM, $100 \mu \mathrm{l}$ milk EVs, or $100 \mu \mathrm{l} \mathrm{EV-depleted} \mathrm{control} \mathrm{for} 5 \mathrm{~h}$. Following culture, cells were harvested and stained with fluorescent conjugated antibodies, which were E-cadherin-PE (clone 67A4, BD Biosciences, dilution 1:50) and EPCAM-APC (EBA1, BD Biosciences, dilution 1:50), or with corresponding isotypes which were PE mouse IgG1 (BD Biosciences, dilution 1:50) or APC mouse IgG1 (BD Biosciences, dilution 1:50). The gating strategy for the characterization of E-cadherin and EPCAM on epithelial cells is indicated in Supplementary Figure 3. Proliferation of purified CD4+ T cells was assessed by labelling cells with 
$2 \mu \mathrm{M}$ CellTrace Violet (Invitrogen) or $0.5 \mu \mathrm{M}$ CFSE (Sigma-Aldrich) prior to culture. Following culture, cells were harvested and stained with fluorescent conjugated antibodies. Antibodies used were: CD3-PE-Cy7 (UCHT1, dilution 1:100), CD4-PerCPCy5.5 (OKT4, dilution 1:50), CD25-Alexa488 (BC96, dilution 1:20), CD45RA-PE (HI100, dilution 1:50), and CD45RO-APCCy7 (UCHL1, dilution 1:50), CD127-PE-Cy7 (A019D5, dilution 1:20) (all from BioLegend). FoxP3-APC (eBioscience, San Diego, CA, USA, 236A/E7, dilution 1:25). Intracellular staining for Treg phenotype were done using the Anti-human Foxp3 Staining Set (eBioscience) according to the manufacturer's protocol. The gating strategy for the characterization Tregs is indicated in Supplementary Figure 12. Cells were measured on a BD Canto II (BD Biosciences), and analyzed by FlowJo (v10.1 FlowJo, Ashland, OR, USA).

\subsection{6 | Functional enrichment, network- and annotation analysis}

Previously, we unravelled the common milk-EV proteome (van Herwijnen et al., 2016), in which 367 proteins were identified in all 7 human milk EV samples tested. These proteins were loaded into the STRING (version 10.5) protein-protein interaction network analysis tool (Szklarczyk et al., 2015), of which 362 proteins matched the STRING database (Supplementary File 3). Enrichment analysis for biological process on these proteins identified $n=784$ significantly enriched GO-terms, of which several linked to the observed in vitro effects of milk EVs. GO-terms were selected that linked to cell cycle (proliferation) and regulation of migration $(n=33)$, or regulation of TLR signalling $(n=26)$, or regulation of T cell activation $(n=48)$ (Supplementary File 3 for data and complete workflow). As enrichment analysis alone does not provide information on the molecular action of a protein, or its position in a signalling cascade, we next performed a comprehensive functional annotation analysis. For that purpose, signalling pathways underlying the observed in vitro effects were constructed using Uniprot entries and the associated sources from Uniprot itself for each individual selected milk EV protein from the enrichment analysis. This pathway analysis was supplemented with a general literature search on both the individual milk EV proteins, as well as the signalling pathways associated to the in vitro observations in order to prevent biased results solely from the enrichment data. Annotation analysis on the selected milk EV proteins was done to determine their interaction with other proteins (either given a common gene name, or a synonym when widely used in literature) and the type of interaction (activating or inhibiting). For each milk EV protein it was decided whether the protein itself, or its cellular interaction partner was relevant to signalling pathways involved in the particular in vitro experiments. Although we could link many milk EV proteins to underlying signalling cascades, yet for some proteins there was insufficient experimental evidence to do so (Supplementary File 3). In the end, all relevant signalling cascades from the full functional annotation analysis are depicted in supplementary figures (Supplementary Figure 2B for proliferation and migration; Supplementary Figure 5B for TLR signalling; Supplementary Figure 13B for T cell activation), in which 'hot spots' were defined as a node in the signalling cascade where a cellular protein would have at least 6 potential interaction partners. Key pathways from the full annotation analysis are shown as a summary in the main figures whereas irrelevant signalling cascades are omitted for clarity. Additionally, protein-protein network analysis on the identified milk EV proteins that linked to the selected GO-terms was performed in STRING (minimum required interaction score set to high confidence 0.700 ), followed by k-means clustering in order to construct clusters of interaction proteins within the network.

\subsection{Statistical analysis}

Data normality was assessed by Shapiro-Wilks test. Normal distributed data were analyzed by paired t-test or one-way ANOVA with Tukey's or Sidak's multiple comparison test. Data that did not have homogenous variances were alternatively tested with Brown-Forsythe and Welch ANOVA and Dunnett's T3 multiple comparison test or Games-Howell's correction, depending on sample size. Figure 3a- $d$ were log-transformed and analyzed in a 2-way mixed model (R-Package lme4 V1.1-26) having EV donor and experiment number as random factors with post-hoc analysis of the least-squares means and performed with Bonferroni multiple comparison correction. Non-normally distributed data were analyzed by Wilcoxon matched-pairs signed rank test or Kruskal-Wallis test with Dunn's multiple comparisons test. GO-analysis in String was tested with a Fisher's exact test followed by a correction for multiple testing. Analyses were performed in GraphPad Prism Software V8.3.1. and R V4.0.3. Significance was defined as ${ }^{\star} P<0.05 ;{ }^{* \star} P<0.01 ;{ }^{* *} P<0.001$ and ${ }^{\star * * \star} P<0.0001$.

\section{ACKNOWLEDGEMENTS}

This work was supported by project 11676 within the framework of a partnership program jointly funded by Nutricia Research and the Dutch Technology Foundation STW, which is part of the Netherlands Organization for Scientific Research (NWO), and is partly funded by the Ministry of Economic Affairs (to Marijke I. Zonneveld; Martijn J.C. van Herwijnen; Frank A. Redegeld; Esther N.M. Nolte-'t Hoen; Marca H.M. Wauben). The European Union's Horizon 2020 Framework Programme under the grant FETOPEN-801367 evFOUNDRY (to Martijn J.C. van Herwijnen; Marije Kleinjan; Marca H.M. Wauben). The European Union's Horizon 2020 research and innovation Programme under the grant Marie Skłodowska-Curie-722148 TRAIN-EV (to Alberta 
Giovanazzi; Marca H.M. Wauben). The TiFN Food and Nutrition; project OH001, 'novel strategies to promote oral health' (to Marcela M. Fernandez-Gutierrez; Michiel Kleerebezem). The European Research Council under the 441 European Union's Seventh Framework Programme (FP/2007-2013)/ERC Grant Agreement number 442337581 (to Esther N.M. Nolte-'t Hoen). The authors are grateful to Bart van der Hee (Host-Microbe Interactomics Group, Wageningen University) for his help with the singlecell tracking analysis of the re-epithelialization image series and Chris A.H. van de Lest for discussions and help on statistical analysis.

\section{AUTHOR CONTRIBUTIONS}

Marca H.M. Wauben, Esther N.M. Nolte-'t Hoen and Frank A. Redegeld conceived and designed the study. Marijke I. Zonneveld, Martijn J.C. van Herwijnen, Marcela M. Fernandez-Gutierrez, Alberta Giovanazzi, Anne Marit de Groot, Marije Kleinjan and Toni M.M. van Capel performed experiments. Marijke I. Zonneveld, Martijn J.C. van Herwijnen, Marcela M. FernandezGutierrez, Alberta Giovanazzi, Anne Marit de Groot, Marije Kleinjan, and Marca H.M. Wauben analyzed the data. Marijke I. Zonneveld, Martijn J.C. van Herwijnen, Marcela M. Fernandez-Gutierrez, Anne Marit de Groot, Alice J.A.M. Sijts, Leonie S. Taams, Esther C. de Jong, Marije Kleinjan, Esther N.M. Nolte-'t Hoen, Marca H.M. Wauben wrote and revised the manuscript. All authors contributed to editing the paper.

\section{REFERENCES}

Admyre, C., Johansson, S. M., Qazi, K. R., Filén, J.-J., Lahesmaa, R., Norman, M., Neve, E. P. A., Scheynius, A., \& Gabrielsson, S. (2007). Exosomes with immune modulatory features are present in human breast milk. Journal of immunology (Baltimore, Md.: 1950) 179, 1969-1978, 179/3/1969 [pii]

Agarwal, V., Bell, G. W., Nam, J-Wu, \& Bartel, D. P. (2015). Predicting effective microRNA target sites in mammalian mRNAs. eLife 4, e05005. https://doi.org/10. 7554/eLife.05005

Ballard, O., \& Morrow, A. L. (2013). Human milk composition: Nutrients and bioactive factors. Pediatric clinics of North America 60, 49-74.

Barth, A. I. M., Kim, H., \& Riedel-Kruse, I. H. (2018). Regulation of epithelial migration by epithelial cell adhesion molecule requires its Claudin-7 interaction domain. Plos One 13, e0204957, https://doi.org/10.1371/journal.pone.0204957

Basha, S., Surendran, N., \& Pichichero, M. (2014). Immune responses in neonates. Expert Review of Clinical Immunology 10, 1171-1184.

Bretz, N. P., Ridinger, J., Rupp, A.-K., Rimbach, K., Keller, S., Rupp, C., Marmé, F., Umansky, L., Umansky, V., Eigenbrod, T., Sammar, M., \& Altevogt, P. (2013). Body fluid exosomes promote secretion of inflammatory cytokines in monocytic cells via Toll-like receptor signaling. The Journal of Biological Chemistry 288, 36691-36702.

Caballero, S., \& Pamer, E. G.(2015). Microbiota-mediated inflammation and antimicrobial defense in the intestine. Annual Review of Immunology 33, $227-256$.

Chatterton, D. E., Nguyen, D. N., Bering, S. B., \& Sangild, P. T. (2013). Anti-inflammatory mechanisms of bioactive milk proteins in the intestine of newborns. The International Journal of Biochemistry \& Cell Biology 45, 1730-1747.

Chen, T., Xie, M.-Y., Sun, J.-J., Ye, R.-S., Cheng, X., Sun, R.-P., Wei, Li-M., Li, M., Lin, De-L., Jiang, Q.-Y., Xi, Q.-Y., \& Zhang, Y.-L. (2016). Porcine milk-derived exosomes promote proliferation of intestinal epithelial cells. Scientific Reports 6, 33862, https://doi.org/10.1038/srep33862

Chung, W. O., \& Dale, B. A. (2008). Differential utilization of nuclear factor-kappaB signaling pathways for gingival epithelial cell responses to oral commensal and pathogenic bacteria. Oral Microbiology and Immunology 23, 119-126.

Cuenda, A., Rouse, J., Doza, Y. N., Meier, R., Cohen, P., Gallagher, T. F., Young, P. R., \& Lee, J. C. (1995). SB 203580 is a specific inhibitor of a MAP kinase homologue which is stimulated by cellular stresses and interleukin-1. Febs Letters 364, 229-233.

Cummins, A. G. (2002). Effect of breast milk and weaning on epithelial growth of the small intestine in humans. Gut 51, 748-754

De Jong, E. C., Vieira, P. L., Kalinski, P., Schuitemaker, J. H. N., Tanaka, Y., Wierenga, E. A., Yazdanbakhsh, M., \& Kapsenberg, M. L. (2002). Microbial compounds selectively induce Th1 cell-promoting or Th2 cell-promoting dendritic cells in vitro with diverse th cell-polarizing signals. Journal of Immunology (Baltimore, Md.: 1950) 168, 1704-1709

Ewald, S. E., Lee, B. L., Lau, L., Wickliffe, K. E., Shi, G.-P., Chapman, H. A., \& Barton, G. M. (2008). The ectodomain of Toll-like receptor 9 is cleaved to generate a functional receptor. Nature 456, 658-662.

Favata, M. F., Horiuchi, K. Y., Manos, E. J., Daulerio, A. J., Stradley, D. A., Feeser, W. S., Van Dyk, D. E., Pitts, W. J., Earl, R. A., Hobbs, F., Copeland, R. A., Magolda, R. L., Scherle, P. A., \& Trzaskos, J. M. (1998). Identification of a novel inhibitor of mitogen-activated protein kinase kinase. Journal of Biological Chemistry 273, 18623-18632.

Fernandez-Gutierrez, M. M., Roosjen, P. P. J., Ultee, E., Agelink, M., Vervoort, J. J. M., Keijser, B., Wells, J. M., \& Kleerebezem, M. (2017). Streptococcus salivarius MS-oral-D6 promotes gingival re-epithelialization in vitro through a secreted serine protease. Scientific Reports 7, 1110-z, https://doi.org/10.1038/s41598-017$11446-\mathrm{Z}$

Fernandez-Gutierrez, M. M., Roosjen, P. P. J., Van Zessen, D. B. H., Wells, J. M., Van Baarlen, P., Stubbs, A. P., \& Kleerebezem, M. (2020). High-Throughput Screening Model to Quantify Re-Epithelialization Kinetics. Protocol Exchange, https://doi.org/10.1038/protex.2019.015

Fernandez-Gutierrez, M. M., Van Zessen, D. B. H., Van Baarlen, P., Kleerebezem, M., \& Stubbs, A. P. (2018). KREAP: An automated Galaxy platform to quantify in vitro re-epithelialization kinetics. Gigascience 7, giy078. https://doi.org/10.1093/gigascience/giy078

Garcia-Cattaneo, A., Gobert, F. - X., Muller, M., Toscano, F., Flores, M., Lescure, A., Del Nery, E., \& Benaroch, P. (2012). Cleavage of Toll-like receptor 3 by cathepsins B and $\mathrm{H}$ is essential for signaling. PNAS 109, 9053-9058.

Gardiner, C., Ferreira, Y. J., Dragovic, R. A., Redman, C. W. G., \& Sargent, I. L. (2013). Extracellular vesicle sizing and enumeration by nanoparticle tracking analysis. Journal of Extracellular Vesicles 2, 19671. https://doi.org/10.3402/jev.v2i0.19671

Gay, N. J., Symmons, M. F., Gangloff, M., \& Bryant, C. E. (2014). Assembly and localization of Toll-like receptor signalling complexes. Nature Reviews Immunology $14,546-558$.

Groeger, S., \& Meyle, J. (2019). Oral Mucosal Epithelial Cells. Frontiers in Immunology 10, 208, https://doi.org/10.3389/fimmu.2019.00208

Harding, F. A., Mcarthur, J. G., Gross, J. A., Raulet, D. H., \& Allison, J. P. (1992). CD28-mediated signalling co-stimulates murine T cells and prevents induction of anergy in T-cell clones. Nature 356, 607-609.

He, Y., Lawlor, N. T., \& Newburg, D. S. (2016). Human milk components modulate toll-like receptor-mediated inflammation. Advances in Nutrition (Bethesda, Md.) 7, 102-111. 
Herwijnen, M. J. C. V., Driedonks, T. A. P., Snoek, B. L., Kroon, A. M. T., Kleinjan, M., Jorritsma, R., Pieterse, C. M. J., Hoen, E. N. M. N-`T., \& Wauben, M. H. M. (2018). Abundantly present miRNAs in milk-derived extracellular vesicles are conserved between mammals. Frontiers Nutritional 5, 81, https: //doi.org/10.3389/fnut.2018.00081

Hock, A., Miyake, H., Li, Bo, Lee, C., Ermini, L., Koike, Y., Chen, Y., Määttänen, P., Zani, A., \& Pierro, A. (2017). Breast milk-derived exosomes promote intestinal epithelial cell growth. Journal of Pediatric Surgery 52, 755-759, S0022-3468(17)30065-9 [pii]

Huang, C. (2004). MAP kinases and cell migration. Journal of Cell Science 117, 4619-4628.

Kondo, T., Kawai, T., \& Akira, S. (2012). Dissecting negative regulation of Toll-like receptor signaling. Trends in Immunology 33, 449-458.

Kumar, S., Jiang, M. S., Adams, J. L., \& Lee, J. C. (1999). Pyridinylimidazole compound SB 203580 inhibits the activity but not the activation of p38 mitogenactivated protein kinase. Biochemical and Biophysical Research Communications 263, 825-831.

Lee, J., Mo, Ji-H., Katakura, K., Alkalay, I., Rucker, A. N., Liu, Yu-T., Lee, H-Ku, Shen, C., Cojocaru, G., Shenouda, S., Kagnoff, M., Eckmann, L., Ben-Neriah, Y., \& Raz, E.(2006). Maintenance of colonic homeostasis by distinctive apical TLR9 signalling in intestinal epithelial cells. Nature Cell Biology 8, $1327-1336$.

Liao, Y., Du, X., Li, J., \& Lonnerdal, B. (2017)Human milk exosomes and their microRNAs survive digestion in vitro and are taken up by human intestinal cells. Molecular Nutrition \& Food Research 61. Epub 201702017 Aug 201700015, https://doi.org/10.1002/mnfr.201700082.

Liew, F. Y., Xu, D., Brint, E. K., \& O’neill, L. A. J. (2005). Negative regulation of toll-like receptor-mediated immune responses. Nature Reviews Immunology 5 , 446-458, nRi1630 [pii]

Lv, Z.-M., Wang, Q., Wan, Q., Lin, J.-G., Hu, M-Si, Liu, Y.-X., \& Wang, R. (2011). The role of the p38 MAPK signaling pathway in high glucose-induced epithelialmesenchymal transition of cultured human renal tubular epithelial cells. Plos One 6, e22806, https://doi.org/10.1371/journal.pone.0022806

Masopust, D., \& Schenkel, J. M. (2013). The integration of T cell migration, differentiation and function. Nature Reviews Immunology 13, 309-320.

Mathieu, M., Martin-Jaular, L., Lavieu, G., \& Théry, C. (2019). Specificities of secretion and uptake of exosomes and other extracellular vesicles for cell-to-cell communication. Nature Cell Biology 21, 9-17.

Mebratu, Y., \& Tesfaigzi, Y. (2009). How ERK1/2 activation controls cell proliferation and cell death: Is subcellular localization the answer? Cell Cycle (Georgetown, Tex.) 8, 1168-1175, 8147 [pii]

Menckeberg, C. L., Hol, J., Simons-Oosterhuis, Y., Raatgeep, H. R. C., De Ruiter, L. F., Lindenbergh-Kortleve, D. J., Korteland-Van Male, A. M., El Aidy, S., Van Lierop, P. P. E., Kleerebezem, M., Groeneweg, M., Kraal, G., Elink-Schuurman, B. E., De Jongste, J. C., Nieuwenhuis, E. E. S., \& Samsom, J. N. (2015). Human buccal epithelium acquires microbial hyporesponsiveness at birth, a role for secretory leukocyte protease inhibitor. Gut 64, 884-893.

Merkenschlager, M., \& Von Boehmer, H. (2010). PI3 kinase signalling blocks Foxp3 expression by sequestering Foxo factors. Journal of Experimental Medicine $207,1347-1350$.

Moutsopoulos, N. M., \& Konkel, J. E. (2018). Tissue-Specific Immunity at the Oral Mucosal Barrier. Trends in Immunology 39, $276-287$.

Raposo, G., \& Stahl, P. D. (2019). Extracellular vesicles: A new communication paradigm? Nature Reviews Molecular Cell Biology 20, 509-510.

Renz, H., Brandtzaeg, P., \& Hornef, M. (2011). The impact of perinatal immune development on mucosal homeostasis and chronic inflammation. Nature Reviews Immunology 12, 9-23.

Robbins, P. D., \& Morelli, A. E. (2014). Regulation of immune responses by extracellular vesicles. Nature Reviews Immunology 14, 195-208.

Sallusto, F., Geginat, J., \& Lanzavecchia, A. (2004). Central memory and effector memory T cell subsets: Function, generation, and maintenance. Annual Review of Immunology 22, 745-763.

Seo, W., Eun, H. S., Kim, So Y., Yi, H.-S., Lee, Y.-S., Park, S.-H., Jang, Mi-J., Jo, E., Kim, S. C., Han, Y.-M., Park, K.-G., \& Jeong, W-II. (2016). Exosome-mediated activation of toll-like receptor 3 in stellate cells stimulates interleukin-17 production by gammadelta T cells in liver fibrosis. Hepatology (Baltimore, Md.) 64, 616-631.

Shamir, E. R., \& Ewald, A. J. (2015).Adhesion in mammary development: Novel roles for E-cadherin in individual and collective cell migration. Current Topics in Developmental Biology 112, 353-382.

Sharma, G.-D., He, J., \& Bazan, H. E. P. (2003). p38 and ERK1/2 coordinate cellular migration and proliferation in epithelial wound healing: Evidence of cross-talk activation between MAP kinase cascades. The Journal of Biological Chemistry 278, 21989-21997.

Steenbeek, S. C., Pham, T. V., Ligt, J., Zomer, A., Knol, J. C., Piersma, S. R., Schelfhorst, T., Huisjes, R., Schiffelers, R. M., Cuppen, E., Jimenez, C. R., \& Rheenen, J. (2018). Cancer cells copy migratory behavior and exchange signaling networks via extracellular vesicles. Embo Journal 37, e98357. https://doi.org/10.15252/ embj. 201798357

Szklarczyk, D., Franceschini, A., Wyder, S., Forslund, K., Heller, D., Huerta-Cepas, J., Simonovic, M., Roth, A., Santos, A., Tsafou, K. P., Kuhn, M., Bork, P., Jensen, L. J., \& Von Mering, C. (2015). STRING v10: Protein-protein interaction networks, integrated over the tree of life. Nucleic Acids Research 43, D447, https://doi.org/10.1093/nar/gku1003

Tinevez, J.-Y., Perry, N., Schindelin, J., Hoopes, G. M., Reynolds, G. D., Laplantine, E., Bednarek, S. Y., Shorte, S. L., \& Eliceiri, K. W. (2017). TrackMate: An open and extensible platform for single-particle tracking. Methods (San Diego, Calif.) 115, 80-90, https://doi.org/10.1016/j.ymeth.2016.09.016

Torregrosa Paredes, P., Gutzeit, C., Johansson, S., Admyre, C., Stenius, F., Alm, J., Scheynius, A., \& Gabrielsson, S. (2014). Differences in exosome populations in human breast milk in relation to allergic sensitization and lifestyle. Allergy 69, 463-471, https://doi.org/10.1111/all.12357

Tuma, P. L., \& Hubbard, A. L. (2003). Transcytosis: Crossing cellular barriers. Physiological Reviews 83, 871-932, https://doi.org/10.1152/physrev.00001.2003

Turfkruyer, M., \& Verhasselt, V. (2015). Breast milk and its impact on maturation of the neonatal immune system. Current Opinion in Infectious Diseases 28, 199-206, https://doi.org/10.1097/QCO.0000000000000165

Turk, B., Turk, V., \& Turk, D. (1997). Structural and functional aspects of papain-like cysteine proteinases and their protein inhibitors. Biological Chemistry 378, 141-150.

Van Den Broek, T., Borghans, J. A. M., \& Van Wijk, F. (2018). The full spectrum of human naive T cells. Nature Reviews Immunology, 18, 363-373.

Van Der Aar, A. M. G., Sibiryak, D. S., Bakdash, G., Van Capel, T. M. M., Van Der Kleij, H. P. M., Opstelten, D.-J. E., Teunissen, M. B. M., Kapsenberg, M. L., \& De Jong, E. C. (2011). Vitamin D3 targets epidermal and dermal dendritic cells for induction of distinct regulatory T cells. The Journal of Allergy and Clinical Immunology 127, 1532-1540.e7.

Van Deun, J., Mestdagh, P., Agostinis, P., Akay, Ö., Anand, S., Anckaert, J., Martinez, Z. A., Baetens, T., Beghein, E., Bertier, L., Berx, G., Boere, J., Boukouris, S., Bremer, M., Buschmann, D., Byrd, J. B., Casert, C., Cheng, L., Cmoch, A., ... Hendrix, A. (2017). EV-TRACK: Transparent reporting and centralizing knowledge in extracellular vesicle research. Nature Methods 14, 228-232.

van Herwijnen, M. J., Zonneveld, Marijke I., Goerdayal, Soenita, Nolte-'t Hoen, Esther N. M., Garssen, Johan, Stahl, Bernd, Altelaar, A. F. Maarten, Redegeld, Frank A., \& Wauben, Marca H. M. (2016). Comprehensive proteomic analysis of human milk-derived extracellular vesicles unveils a novel functional proteome distinct from other milk components. Molecular \& Cellular Proteomics: MCP 15, 3412-3423, M116.060426 [pii]

Victora, C. G., Bahl, R., Barros, A. J. D., França, G. V. A., Horton, S., Krasevec, J., Murch, S., Sankar, M. J., Walker, N., \& Rollins, N. C. (2016). Breastfeeding in the 21st century: Epidemiology, mechanisms, and lifelong effect. Lancet (London, England) 387, 475-490. 
Ye, W., Tang, X., Yang, Z., Liu, C., Zhang, X., Jin, J., \& Lyu, J. (2017). Plasma-derived exosomes contribute to inflammation via the TLR9-NF-kappaB pathway in chronic heart failure patients. Molecular Immunology $87,114-121$ [pii]

Yu, S., Zhao, Z., Sun, L., \& Li, P. (2017). Fermentation results in quantitative changes in milk-derived exosomes and different effects on cell growth and survival. Journal of Agricultural and Food Chemistry 65, 1220-1228.

Zohn, I. E., Li, Y., Skolnik, E. Y., Anderson, K. V., Han, J., \& Niswander, L. (2006). p38 and a p38-interacting protein are critical for downregulation of E-cadherin during mouse gastrulation. Cell 125, 957-969.

Zonneveld, M. I., Brisson, Alain R., van Herwijnen, Martijn J. C., Tan, Sisareuth, van de Lest, Chris H. A., Redegeld, Frank A., Garssen, Johan, Wauben, Marca H. M., \& Nolte-'t Hoen, Esther N. M. (2014). Recovery of extracellular vesicles from human breast milk is influenced by sample collection and vesicle isolation procedures. Journal of Extracellular Vesicles 3. eCollection 22014, https://doi.org/10.3402/jev.v3.24215.

\section{SUPPORTING INFORMATION}

Additional supporting information may be found online in the Supporting Information section at the end of the article.

How to cite this article: Zonneveld MI, van Herwijnen MJC, Fernandez-Gutierrez MM, et al. Human milk extracellular vesicles target nodes in interconnected signalling pathways that enhance oral epithelial barrier function and dampen immune responses. J Extracell Vesicles. 2021;10:e12071. https://doi.org/10.1002/jev2.12071 